\title{
Emotional expressions associated with therapeutic inertia in multiple sclerosis care
}

\author{
Saposnik, Gustavo ; Oh, Jiwon ; Terzaghi, Maria A ; Kostyrko, Pawel ; Bakdache, Fabien ; Montoya,
} Alonso ; Jaja, Blessing N R ; Nisenbaum, Rosane ; Ruff, Christian C ; Tobler, Philippe N

\begin{abstract}
Background Emotions play a critical role in our daily decisions. However, it remains unclear how and what sort of emotional expressions are associated with therapeutic decisions in multiple sclerosis (MS) care. Our goal was to evaluate the relationship between emotions and affective states (as captured by muscle facial activity and emotional expressions) and TI amongst neurologists caring for MS patients when making therapeutic decisions. Methods 38 neurologists with expertise in MS were invited to participate in a face-to-face study across Canada. Participants answered questions regarding their clinical practice, aversion to ambiguity, and the management of 10 simulated case-scenarios. TI was defined as lack of treatment initiation or escalation when there was clear evidence of clinical and radiological disease activity. We recorded facial muscle activations and their associated emotional expressions during the study, while participants made therapeutic choices. We used a validated machine learning algorithm of the AFFDEX software to code for facial muscle activations and a predefined mapping to emotional expressions (disgust, fear, surprise, etc.). Mixed effects models and mediation analyses were used to evaluate the relationship between ambiguity aversion, facial muscle activity/emotional expressions and TI measured as a binary variable and a continuous score. Results 34 (89.4\%) neurologists completed the study. The mean age [standard deviation (SD)] was 44.6 (11.5) years; $38.3 \%$ were female and $58.8 \%$ self-identified as MS specialists. Overall, 17 (50\%) participants showed TI in at least one case-scenario and the mean (SD) TI score was $0.74(0.90)$. Nineteen $(55.9 \%)$ participants had aversion to ambiguity in the financial domain. The multivariate analysis adjusted for age, sex and MS expertise showed that aversion to ambiguity in the financial domain (OR 1.56, 95\% CI 1.32-1.86) was associated with TI. Most common muscle activations included mouth open (23.4\%), brow furrow (20.9\%), brow raise (17.6\%), and eye widening (13.1\%). Most common emotional expressions included fear (5.1\%), disgust (3.2\%), sadness $(2.9 \%)$, and surprise $(2.8 \%)$. After adjustment for age, sex, and physicians' expertise, the multivariate analysis revealed that brow furrow (OR 1.04; 95\% CI 1.003-1.09) and lip suck (OR 1.06; 95\% CI 1.01-1.11) were associated with an increase in TI prevalence, whereas upper lip raise (OR 0.30; 95\% CI 0.15-0.59), and chin raise (OR 0.90; 95\% CI 0.83-0.98) were associated with lower likelihood of TI. Disgust and surprise were associated with a lower TI score (disgust: $\mathrm{p}<0.001$; surprise: $\mathrm{p}=0.008$ ) and lower prevalence of TI (ORdisgust: 0.14 , 95\%CI 0.03-0.65; ORsurprise: 0.66, 94\%CI 0.47-0.92) after adjusting for covariates. The mediation analysis showed that brow furrow was a partial mediator explaining $21.2 \%$ (95\% CI 14.9\%-38.9\%) of the association between aversion to ambiguity and TI score, followed by nose wrinkle $12.8 \%(95 \%$ CI $8.9 \%$ $23.4 \%)$. Similarly, disgust was the single emotional expression (partial mediator) that attenuated $(-13.2 \%$, $95 \%$ CI $-9.2 \%$ to $-24.3 \%$ ) the effect of aversion to ambiguity on TI. Conclusions TI was observed in half of participants in at least one case-scenario. Our data suggest that facial metrics (e.g. brow furrow, nose wrinkle) and emotional expressions (e.g. disgust) are associated with physicians' choices and partially mediate the effect of aversion to ambiguity on TI.
\end{abstract}

DOI: https://doi.org/10.1016/j.msard.2019.05.029 
Posted at the Zurich Open Repository and Archive, University of Zurich

ZORA URL: https://doi.org/10.5167/uzh-175026

Journal Article

Accepted Version

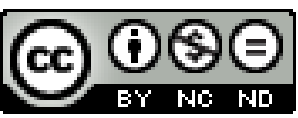

The following work is licensed under a Creative Commons: Attribution-NonCommercial-NoDerivatives 4.0 International (CC BY-NC-ND 4.0) License.

Originally published at:

Saposnik, Gustavo; Oh, Jiwon; Terzaghi, Maria A; Kostyrko, Pawel; Bakdache, Fabien; Montoya, Alonso; Jaja, Blessing N R; Nisenbaum, Rosane; Ruff, Christian C; Tobler, Philippe N (2019). Emotional expressions associated with therapeutic inertia in multiple sclerosis care. Multiple Sclerosis and Related Disorders, 34:17-28.

DOI: https://doi.org/10.1016/j.msard.2019.05.029 


\title{
Emotional Expressions associated with Therapeutic Inertia
}

\section{in Multiple Sclerosis Care}

\author{
Authors: Gustavo Saposnik, MD ${ }^{1-4}$, Jiwon Oh, MD PhD ${ }^{1,4}$, Maria A. Terzaghi, Pharm ${ }^{1,4}$, Pawel Kostyrko, \\ MD¹, Fabien Bakdache, PharmD ${ }^{5}$, Alonso Montoya, $\mathrm{MD}^{5}$, Blessing N.R. Jaja, MD PhD ${ }^{3}$, Rosane \\ Nisenbaum, $\mathrm{PhD}^{4,6}$, Christian C. Ruff, $\mathrm{PhD}^{2}$, Philippe N. Tobler, $\mathrm{PhD}^{2}$ \\ ${ }^{1}$ Division of Neurology, Department of Medicine, St. Michael's Hospital, University of Toronto; Canada \\ ${ }^{2}$ Laboratory for Social and Neural Systems Research, Department of Economics, University of Zurich; Switzerland \\ ${ }^{3}$ Decision Neuroscience Unit, St Michael's Hospital, University of Toronto \\ ${ }^{4}$ Li Ka Shing Knowledge Institute, St. Michael's Hospital, University of Toronto; Canada \\ ${ }^{5}$ Medical Affairs, Neuroscience, Hoffmann-La Roche, Mississauga; Canada \\ ${ }^{6}$ Applied Health Research Centre, Centre for Urban Health Solutions, St Michael's Hospital, Toronto; Canada
}

Title character count: 75

Number of references: 62

Number of tables: 2

Number of figures: 5

Word count abstract: 499

Word count paper: 4210

\section{Corresponding author:}

Dr. Gustavo Saposnik

PhD Candidate in Neuroeconomics

University of Zurich

Associate Professor, Department of Medicine (Neurology)

St. Michael's Hospital, University of Toronto

55 Queen St E, Toronto, Ontario, M5C 1R6

Tel: (416) 864-5155, Fax: (416) 864-5150

Email: saposnikg@smh.ca

Search terms: multiple sclerosis, disease-modifying therapy, therapeutic inertia, neuroeconomics, decision making, risk 


\section{Authors Disclosures:}

Dr. Gustavo Saposnik is supported by the Heart and Stroke Foundation Career Scientist Awards following an open peer-reviewed competition.

Dr. Alonso Montoya, Fabien Bakdache are employees at the Department of Medical Affairs, Neuroscience, Hoffmann-La Roche Limited, Mississauga, ON, Canada

Maria Terzaghi, Blessing Jaja, Rosane Nisenbaum - no disclosures

Dr. Oh has received research funding from the MS Society of Canada, the National MS Society, Brain Canada, Sanofi-Genzyme, Roche, and Biogen. Dr. Oh has received personal compensation for consulting or speaking from EMD-Serono, Genzyme, Biogen, Novartis, Celgene, and Roche.

Prof. Ruff received research funding from the Swiss National Science Foundation (100019L_173248)

Prof. Philippe Tobler received research funding from the Swiss National Science Foundation (PP00P1_150739 and 100014_165884) and Pfizer.

Study funding: The study was sponsored by Division of Neurology at the University of Toronto with the collaboration of Neuro-sens and funded through a research collaboration agreement with Roche Canada. The sponsoring entities were not involved in the design, execution, analysis, and interpretation or reporting of the results. Ocrelizumab (manufactured by Roche) was not included as a therapeutic option in any of the casescenarios.

\section{Authors contributions:}

Gustavo Saposnik: study concept and design, acquisition of data, analysis and interpretation of the data and obtaining funding.

Jiwon Oh: supervision of MS case-scenarios, interpretation of the data, critical revision of the manuscript for intellectual content.

Maria Terzaghi: study implementation, interpretation of the data, and critical revision of the manuscript for intellectual content.

Fabien Bakdache: interpretation of the data, critical revision of the manuscript for intellectual content.

Alonso Montoya: interpretation of the data, critical revision of the manuscript for intellectual content.

Blessing Jaja: analysis and critical revision of the manuscript for intellectual content.

Rosane Nisenbaum: supervision of statistical analysis, critical revision of the manuscript for intellectual content.

Prof. Ruff: interpretation of the data and critical revision of the manuscript for intellectual content. 
Prof. Tobler: interpretation of the data and critical revision of the manuscript for intellectual content.

\section{Acknowledgements}

The authors are most grateful to all physicians participating in the study.

We appreciate the support from the Department of Economics at the University of Zurich by facilitating access to the Qualtrics platform. 


\section{Abstract:}

Background: Emotions play a critical role in our daily decisions. However, it remains unclear how and what sort of emotional expressions are associated with therapeutic decisions in multiple sclerosis (MS) care.

Our goal was to evaluate the relationship between emotions and affective states (as captured by muscle facial activity and emotional expressions) and TI amongst neurologists caring for MS patients when making therapeutic decisions.

Methods: 38 neurologists with expertise in MS were invited to participate in a face-to-face study across Canada. Participants answered questions regarding their clinical practice, aversion to ambiguity, and the management of 10 simulated case-scenarios. TI was defined as lack of treatment initiation or escalation when there was clear evidence of clinical and radiological disease activity. We recorded facial muscle activations and their associated emotional expressions during the study, while participants made therapeutic choices. We used a validated machine learning algorithm of the AFFDEX software to code for facial muscle activations and a predefined mapping to emotional expressions (disgust, fear, surprise, etc.). Mixed effects models and mediation analyses were used to evaluate the relationship between ambiguity aversion, facial muscle activity/emotional expressions and TI measured as a binary variable and a continuous score.

Results: 34 (89.4\%) neurologists completed the study. The mean age [standard deviation (SD)] was 44.6 (11.5) years; $38.3 \%$ were female and $58.8 \%$ self-identified as MS specialists. Overall, 17 (50\%) participants showed TI in at least one case-scenario and the mean (SD) TI score was 0.74 (0.90). Nineteen (55.9\%) participants had aversion to ambiguity in the financial domain. The multivariate analysis adjusted for age, sex and MS expertise showed that aversion to ambiguity in the financial domain (OR $1.56,95 \% \mathrm{Cl} 1.32-1.86$ ) was associated with TI. Most common muscle activations included mouth open (23.4\%), brow furrow (20.9\%), brow raise (17.6\%), and eye widening (13.1\%). Most common emotional expressions included fear (5.1\%), disgust (3.2\%), sadness (2.9\%), and surprise (2.8\%). After adjustment for age, sex, and physicians' expertise, the multivariate analysis revealed that brow furrow (OR 1.04; 95\%Cl 1.003-1.09) and lip suck (OR 1.06; 95\% Cl 1.01-1.11) were associated with an increase in TI prevalence, whereas upper lip raise (OR 0.30; 95\% $\mathrm{Cl} 0.15-0.59)$, and chin raise (OR $0.90 ; 95 \% \mathrm{Cl}$ 0.83-0.98) were associated with lower likelihood of TI. Disgust and surprise were associated with a lower TI score (disgust: $p<0.001$; surprise: $\mathrm{p}=0.008$ ) and lower prevalence of $\mathrm{TI}\left(\mathrm{OR}_{\text {disgust }}\right.$ : $0.14,95 \% \mathrm{Cl} 0.03-0.65$; OR surprise: 0.66 , $94 \% \mathrm{Cl}$ 0.47-0.92) after adjusting for covariates.

The mediation analysis showed that brow furrow was a partial mediator explaining $21.2 \%(95 \% \mathrm{Cl} 14.9 \%-38.9 \%)$ of the association between aversion to ambiguity and $\mathrm{TI}$ score, followed by nose wrinkle $12.8 \%(95 \% \mathrm{Cl} 8.9 \%$ - 
23.4\%). Similarly, disgust was the single emotional expression (partial mediator) that attenuated $(-13.2 \%, 95 \% \mathrm{Cl}$ $-9.2 \%$ to $-24.3 \%$ ) the effect of aversion to ambiguity on $\mathrm{TI}$.

Conclusions: TI was observed in half of participants in at least one case-scenario. Our data suggest that facial metrics (e.g. brow furrow, nose wrinkle) and emotional expressions (e.g. disgust) are associated with physicians' choices and partially mediate the effect of aversion to ambiguity on TI. 


\section{Background:}

The role of emotions in decision-making has been investigated for decades. Recent studies have shown that emotions are the dominant driver of the majority of meaningful goal-directed decisions in life.(1, 2). Different emotions (fear, disgust, stress, surprise, etc.) manifested by facial muscle activation can modulate our perceptions and valuation of individual choices by activating different pathways involving the striatum, orbitofrontal cortex, the medial and dorsolateral prefrontal cortex, the inferior parietal cortex, the amygdala and/or insular cortex.(3-5)

Previous studies have shown that decision making was associated with muscle activation (e.g. brow furrow, brow raise, lip pucker, mouth opening) and emotions (e.g. fear, sadness, anger, surprise) in consumers or healthy volunteers. $(3,6,7)$ For example, fear appears to be associated with pessimistic risk assessments and risk-averse choices, whereas anger can provoke an optimistic estimations of risk and risk-seeking behavior.(6-8) Similarly, some emotions, such as anger, surprise, and optimism are associated with participants' tolerance to ambiguity and the selection of optimal choices. $(9,10)$ However, limited information is available regarding how facial muscle activity (and derived emotional expressions) relate to physicians' therapeutic decisions.

Therapeutic inertia ( $\mathrm{TI}$ ) is a term that was introduced in 2006 to define the absence of treatment initiation or intensification when treatment goals are unmet.(11-13) $\mathrm{Tl}$ is a common phenomenon affecting $50 \%$ to $90 \%$ of doctors caring for patients with chronic conditions (e.g. hypertension, diabetes, multiple sclerosis) and leading to poorer clinical outcomes and higher health care costs.(11-14) Previous studies have identified factors associated with $\mathrm{TI}(15,16)$, and physician factors (e.g. aversion to ambiguity) are considered the main contributors.(16-18). To our knowledge, there are no data showing a relationship between facial muscle activation, emotional expressions, and therapeutic decisions under uncertainty (or ambiguity) among practicing physicians.

In this study, we evaluated facial muscle activation (and emotional expression) associated with therapeutic choices, particularly TI. We also sought to evaluate the mediation effect between a physical (e.g. facial muscle 
activity) or emotional (fear, disgust, surprise) response with a therapeutic decision. Given the known associations between specific facial muscle activation and emotional expression (anger, fear, disgust, surprise, etc.) with an increased attention response that precedes participants' choices $(10,19,20)$, we hypothesized that facial muscle activity (e.g. upper lip raise) and emotional expression (disgust, surprise) would increase participants' awareness and therefore mediating the relationship between aversion to ambiguity and TI. We assessed emotional expressions amongst physicians who care for people living with multiple sclerosis (MS) as this care model is representative of the paradigm of complex therapeutic decisions (e.g. multiple therapeutic options with a broad therapeutic range- e.g. different safety and efficacy profiles) in the management of a chronic medical condition.

\section{METHODS:}

\section{Study design}

We conducted a cross-sectional study using the online platform Qualtrics. The study included 10 MS casevignettes to evaluate $\mathrm{TI}$ and 2 behavioural experiments to determine subject's attitudes towards ambiguity. Case-scenarios were designed by our research team and MS experts (JO, GS). Overall, 8 cases aimed to assess appropriate escalation of treatment (whereby an absence of treatment change corresponds to TI), while the remaining 2 cases were designed as controls (no indication for treatment escalation as there was no evidence of a clinical relapse and disease activity on brain imaging). After completing demographic information and questions regarding their current clinical practices, participants were exposed to behavioral experiments assessing ambiguity aversion and then responded to case-scenarios (Figure 1).

Behavioural experiments were designed to assess aversion to ambiguity in the health and financial domains as previously reported by our group. $(18,21,22)$ Ambiguity aversion is defined as dislike for events with unknown probability over events with known probability.(23) For example, an ambiguity-averse individual would rather choose a treatment where the probability of benefits or side effects are known (even if these are somewhat unfavourable) over one where these probabilities are unknown. Specifically, in the health domain, participants 
were asked to choose between Treatment A (50\% probability of survival) or "Treatment B" (the probability of survival is unknown). In the financial domain, participants were asked to choose between a visual option with known $50 / 50$ probability of winning 400 or 0 US\$ versus an option with unknown probability of the same outcomes. In both domains, we used grey bars to represent five levels $(10 \%, 30 \%, 50 \%, 70 \%$, and $90 \%)$ of unknown probability. Aversion to ambiguity was indexed in two-ways: binary (preference for the known probability in all 5 levels) and as continuous variable (number of levels that participants selected the known probability over 5). Details of the protocol and case-scenarios were previously published.(18, 24, 25)

Participants: Practicing neurologists actively involved in the care of people living with MS from across Canada were invited to participate in our study by the Canadian Network of MS Clinics and Neuro-sens (Neurosens.com). These networks capture most of these neurologists in Canada. Participants were recruited from December 13, 2017 to March 2, 2018. Physicians whose practice focuses primarily on caring for MS patients were classified as 'MS specialists'.

The study was conducted in an ambulatory clinic-type setting to mimic the current clinical environment. Room temperature, light conditions, and participants' sitting positions were standardized. We used a high definition webcam (Logitech Pro 920○) to capture facial movements. All participants had at least 90\% muscle detection by the camera during the study period. Facial detection algorithms from AFFDEX (see below) were integrated with the Qualtrics survey platform through iMotions software (iMotions.com). The mean (median) duration of the study was 44.9 (39.9) minutes. Participants were compensated with 400 Canadian dollars. Written informed consent was obtained from all participants. The study was approved by the Research Ethics Board of St. Michael's Hospital, University of Toronto, Canada.

\section{Assessment of emotional expressions:}

We used AFFDEX, a machine learning algorithm software that detects for emotional expressions based on facial muscle activity. $(19,26)$ AFFDEX has been validated in more than 6 million facial videos from over 87 countries showing an excellent accuracy (area under the curve greater than 0.9) (https://www.affectiva.com/how/how- 
it-works/, accessed Feb 28, 2019). This algorithm uses different features to identify 34 facial landmarks (e.g. eye corners, eye centers, nose tip, mouth corner) with a threshold area, discarding background regions (Figure 2). The region of interest (ROI) contains the whole face including eyes, mouth and nose. AFFDEX applies distinct analytical procedures to identify emotional expressions (https://developer.affectiva.com/mapping-expressionsto-emotions/). During our study, facial detection was recorded to analyze each video frame. Eye blinking and closure were filtered-out. AFFDEX uses frames with a positive detection for the subsequent analysis.

Facial muscle activity is the main unit of study in emotional expressions. Facial movements are detected and mapped on probability values of emotional states (e.g. sadness, joy, disgust, anger, surprise, fear, contempt). The probabilities returned by the AFFDEX module range between zero and one. A value of zero indicates no evidence and a value of one the highest evidence that a certain emotion is fully expressed.(26) We used raw values of each individual's facial expression to directly compare amongst participants. This approach mitigates potential errors in the algorithms created to represent emotional expressions due to lack of matching with predefined facial muscle activity.

We use a proxy measure of participants' arousal by combining the level of attention (a summary measure of the time frame each participant was looking at the screen) and engagement (a weighted sum of facial expressions). We compared facial muscle activity and emotional expressions between participants with and without TI.

Multiple Sclerosis and Definitions: In the context of MS, TI is defined as the lack of treatment initiation or escalation when there is evidence of disease activity, based on clinical evidence and neuroimaging markers.(21, 27, 28) A more proactive management strategy, including earlier use of high-efficacy DMTs and close monitoring of the clinical and radiological response to treatment is recommended to slow the progression of physical and cognitive impairments in patients with relapsing-remitting multiple sclerosis (RRMS).(29-31) Early treatment escalation has been shown to reduce relapse rates, disability progression, and MRI activity. $(32,33)$ For the primary analysis, we used an accepted definition of disease activity that would prompt treatment initiation or escalation. $(27,34,35)$ Disease activity was defined as the presence of a clinical relapse plus the presence of 
more than four new brain lesions in follow-up magnetic resonance imaging (MRI) scans or at least one gadolinium-enhancing lesion.(34, 35).

TI was measured as both a continuous score and as a binary variable. The TI score corresponded to the number of case-scenarios where treatment initiation or escalation was warranted but not provided (numerator) divided by the total number of case-scenarios where $\mathrm{TI}$ could occur (denominator; $\mathrm{n}=8$ ). TI as a binary variable (presence/absence) was determined as the lack of treatment initiation or escalation given disease activity in at least one case-scenario.

Outcome measures: The primary outcomes of the study was the association between facial muscle activity and inferred emotional expression of participants at stimulus presentation (audio introducing MS case-scenarios under uncertainty) when making therapeutic choices and TI.

\section{Statistical analysis:}

We utilized two analytical approaches: i) a descriptive assessment of facial muscle activation and emotional expressions, and ii) a mediation analysis to assess how the association between aversion to ambiguity and $\mathrm{TI}$ may be mediated by facial activations and emotional expressions. Mediation analysis is a technique commonly used in social sciences and consumer research to make causal inferences about the influence of specific factors (e.g. demographic variables, participant's characteristics, etc.) on an outcome via a third variable (called 'mediator').(36, 37) A mediator is a variable that modulates the relationship between that factor with the outcome of interest. $(36,37)$ In our analysis, the independent variable was aversion to ambiguity and the dependent variable was therapeutic inertia. Facial muscle activity or an emotional expression were individually included as mediators. Further details are illustrated in the appendix (Figures e1 and e2).

The primary analysis was a descriptive assessment of the presence of facial muscle activation and emotional expression among participants with and without TI (binary) and by the TI score. For each screen face by participants, we calculated the percentage of the frames in which each facial muscle was detected relative to 
the total number of available frames as part of the AFFDEX software. Then, we identified the time period of the stimulus presentation and the time period of participants' responses when making therapeutic decisions to specifically evaluate the association between facial muscle activation and emotional expressions during these two critical events. Finally, we compared the percentages of facial muscle activation and emotional expressions between participants with and without TI and related them to the TI score. We used a proxy measure of participants' arousal defined as a summary score between attention (range 0-100) and engagement (range 0100).

Specifically, we used mixed effects logistic and linear models to assess relationships between $\mathrm{TI}$ (and TI score) and the percentage of facial muscle movements (and emotional expressions) accounting for clustering (repeated observations on participants). The analysis was adjusted for the following explanatory variables: age, sex, specialist status (MS expert vs. general neurologists). Practice setting (academic vs non-academic), percentage of time devoted to clinical care, and number of MS patients assessed per week had no significant impact on the association between emotional expressions and TI.

We previously found an association between aversion to ambiguity and increased prevalence of TI (18). Here, we aimed to replicate this association and evaluated whether this observed association is mediated by facial muscle activation or emotional expression. For the mediation analysis we used the STATA command 'medeff' (see details of the models in the Appendix).(38, 39) We also use structural equation modeling (SEM) to graphically represent the estimated mediation effects of facial metrics or emotional expressions (see details and interpretation of graphs in the Appendix).(40)

In a sensitivity analysis, we considered the effect of adding participants' number of MS patients seen per week, practice type (academic vs. non-academic), or years of practice instead of participants' expertise in the multivariate models.

Goodness of fit was assessed by the c-statistic for TI (binary outcome) and R-squared for the TI score. All tests were 2-tailed, and p-values $<0.05$ were considered significant. We used STATA 13 (College Station, TX: StataCorp LP) to conduct all analyses. 
To facilitate the interpretation of findings, we performed the following four analyses:

1) We evaluated the prevalence of facial muscle activations and emotional expressions; 2) We examined their association with the likelihood of $\mathrm{TI}$ and the $\mathrm{TI}$ score; 3) We assessed the relationship between facial metrics and emotional expression with ambiguity aversion (main predictor of $\mathrm{Tl}$ in our previous studies)(18); and 4) we conducted a mediation analysis to determine whether facial muscle activation or emotional expression modulate the relationship between the aversion to ambiguity (independent variable) and TI (outcome).

\section{RESULTS:}

\section{Participant characteristics:}

Of the 38 neurologists who were invited to participate in the emotional recognition study, 34 cooperated (cooperation rate: $89.5 \%$ ) and 34 (completion rate: $100 \%$ ) completed the study. The mean age (SD) of study participants was 44.6 ( \pm 11.6 ) years; 13 participants (38.2\%) were female. Twenty participants (58.8\%) primarily focused their practice on MS care. Participants had on average $12.5( \pm 12)$ years of experience and assessed 23.1 ( \pm 16$)$ MS patients per week. Table 1 summarizes baseline characteristics of the study population.

TI was present in $50.0 \%$ of participants in at least one case-scenario. The mean $\mathrm{TI}$ score was $0.74( \pm 0.90)$, and the range was 0 to 3 .

\section{1) Prevalence of facial or emotional expressions:}

The most commonly observed muscle activations included: mouth open (23.4\%), brow furrow (20.9\%), brow raise (17.6\%), and eye widening (13.1\%) (Figure $2 A)$. Brow furrow was associated with $\mathrm{TI}(\mathrm{p}<0.001)$. The most commonly decoded emotional expressions included: fear (5.1\%), disgust (3.2\%), sadness (2.9\%), and surprise (2.8\%) (Figure 2C). Differences in facial muscle activation and emotional expressions by TI status are represented in Figure 2B and 2D.

Participants with muscle facial activations and emotional expressions had higher arousal scores. For example, arousal scores were significantly higher among participants with disgust (180.7 vs. 133.1; $p=0.04)$, surprise (77.6 
vs. $122.4 ; p=0.02$ ), fear (189.9 vs $131.6 ; p=0.02$ ). Similar findings were observed for facial muscle activations associated with $\mathrm{TI}$ (e.g. brow furrow [p<0.001], brow raise $[p<0.001]$, lip suck $[p<0.001]$, mouth open $[p=0.02]$, nose wrinkle $[p<0.01])$.

\section{2) Basic associations between $\mathrm{TI}$ and facial or emotional expressions:}

The multivariate mixed effects logistic regression after adjustment for age, sex, and physicians' expertise revealed that brow furrow (OR 1.04; 95\% $\mathrm{Cl} 1.003-1.09$ ) and lip suck (OR 1.06; 95\% Cl 1.01-1.11) were associated with an increase in $\mathrm{TI}$ prevalence, whereas upper lip raise (OR 0.30; 95\% Cl 0.15-0.59), chin raise $(\mathrm{OR} 0.90 ; 95 \% \mathrm{Cl}$ 0.83-0.98), and nose wrinkle (OR 0.08; 95\% Cl 0.007-0.97) were associated with lower likelihood of TI (c-statistic: 0.889). Similar findings were obtained with linear mixed models (brow furrow: $p=0.05$; lip suck: $p<0.001$; nose wrinkle: $p=0.017$, upper lip raise: $p<0.001$; chin raise: $p<0.001$; $R$-squared: 0.373 ) where the $\mathrm{TI}$ score as the outcome of interest.

In the emotional expression analysis, the presence of disgust (characterized by nose-wrinkle and upper lip raise) and surprise (characterized increased brow raise and decrease brow furrow) were associated with lower prevalence of TI scores (disgust: $p<0.001$; surprise: $p=0.008)$ and $\mathrm{TI}\left(O R_{\text {disgust }}: 0.14,95 \% \mathrm{Cl} 0.03-0.65\right.$; $O R_{\text {surprise: }}$ : $0.66,94 \% \mathrm{Cl} 0.47-0.92$ ) after adjusting for age, sex and physicians' expertise. Fear was not associated with either $\mathrm{TI}\left(\mathrm{OR}_{\text {fear: }} \mathbf{0 . 3 7}, 95 \% \mathrm{Cl} 0.03-5.43\right)$ or the TI score $(p=0.14)$.

\section{3) Relation between facial and emotional expressions to ambiguity aversion:}

In our previous studies, aversion to ambiguity in the financial domain was the most relevant predictor of $\mathrm{TI}(18)$. Similarly, in the present study, 19 (55.9\%) participants never chose an ambiguous alternative in the financial domain and $11(32.4 \%)$ in the health domain. The multivariate analysis adjusted for age, sex and MS expertise showed that aversion to ambiguity in the financial (OR $1.56,95 \% \mathrm{Cl} 1.32-1.86)$ and health $(\mathrm{OR} 1.12,95 \% \mathrm{Cl} 1.02-$ 1.22) domains were independent predictors of $\mathrm{TI}$ (Table 2). Similarly, for every $20 \%$ increase in the degree of ambiguity, there was a $21.5 \%$ increment $(95 \% \mathrm{Cl} 3.0 \%-40.0 \%)$ in the $\mathrm{TI}$ score. 
Given the consistent association between aversion to ambiguity and $\mathrm{TI}$ in this study and prior studies, we also explored the association between facial muscle activity and emotional expression and ambiguity aversion. The multivariate analysis revealed that mouth opening (OR 2.10,95\% $\mathrm{Cl} 1.35-3.26 ; p=0.001$ ), brow furrow (OR 2.93, 95\% Cl 1.84-4.65; $p=<0.001)$, chin raise (OR 3.16, 95\%Cl 1.51-6.62; $p=0.002)$, and lip suck (OR 0.38, 95\%Cl $0.21-$ $0.70 ; p=0.002$ ) were the facial muscle activations associated with higher aversion to ambiguity in the financial domain. Disgust (OR 0.22, 95\% $\mathrm{Cl} 0.08-0.65 ; \mathrm{p}=0.006$ ) was the single emotional expression associated with lower aversion to ambiguity.

The mixed linear regression analysis adjusted for age, sex, participants' expertise, emotional expression (disgust) or facial muscle activation (brow furrow, mouth opening, and lip suck) and degree of aversion to ambiguity in the financial domain is presented in Figure 3. For every $20 \%$ increase in the degree of ambiguity (e.g. from $10 \%$ to $30 \%, 30 \%$ to $50 \%$ and so on), there was a $21.5 \%$ increment $(95 \% \mathrm{Cl} 16.9 \%-26.0 \%)$ in the $\mathrm{TI}$ score (R-squared 0.35) (Figure 3A). Nearly identical results were observed when the linear mixed model included facial muscle activations instead of emotional expression (R-squared 0.38) (Figure 3B). In contrast, there were no associations between facial muscle activation and emotional expression with aversion to ambiguity in the health domain.

\section{4) Mediation analysis: facial and emotional expressions modulate the relationship between aversion to} ambiguity and $\mathrm{TI}$

We found that brow furrow followed by nose wrinkle were the strongest mediators, respectively explaining $21.2 \%(95 \% \mathrm{Cl} 14.9 \%-38.9 \%)$ and $12.8 \%(95 \% \mathrm{Cl} 8.9 \%-23.4 \%)$ of the effect of aversion to ambiguity in the financial domain on TI (Figures 4A and 4B). Similarly, disgust was the single emotional expression that attenuated (-13.2\%, $95 \% \mathrm{Cl}-9.2 \%$ to $-24.3 \%$ ) the effect of aversion to ambiguity in the financial domain on $\mathrm{TI}$ (Figure $4 \mathrm{C}$ ).

Notably, the direct effect of ambiguity aversion on TI was greater than the indirect effect mediated by brow furrow, nose wrinkle, or disgust (partial mediators). For example, there was a significant, but modest increment $(<5 \%)$ in the R-square values when adding the facial or emotional variable into the mixed models. This is also 
reflected in the larger $\beta$ coefficients for the direct effect between ambiguity aversion and $\mathrm{TI}$ compared to the multiplication of $\beta$ coefficients for the indirect effect (Figure 4 and figure e2).

Other facial muscle activations (e.g. lip suck: p-value 0.84) and emotional expressions had a non-significant or a negligible effect (e.g. surprise and fear $<3 \%$ ). The sensitivity analysis revealed no changes in the $\beta$ coefficients for ambiguity aversion when adjusting mixed models for other covariates (see appendix, figures e3 and e4).

\section{DISCUSSION:}

The influence of emotions on the therapeutic decisions of physicians is an important but largely unexplored field. In the present study, we analyzed facial muscle activations and emotional expressions among neurologists while they were making therapeutic decisions. By using the paradigm of complex therapeutic decisions in MS care, we found that emotional expressions (e.g. disgust and surprise) were associated with lower TI. We also observed that facial components of emotional expressions were also associated with TI. Specifically, brow furrow, lip suck and nose wrinkle were associated with an increased prevalence of $\mathrm{TI}$, whereas upper lip raise, and chin raise were associated with a lower likelihood of TI. We also found that aversion to ambiguity increased the likelihood of TI. Participants with the aforementioned emotional expressions and muscle facial activations had higher arousal scores compared to those without.

The mediation analysis revealed that disgust was the single emotion that attenuated the effect of aversion to ambiguity in the financial domain on TI. Similarly, the assessment of component processes that mapped to emotional expressions revealed that brow furrow and nose wrinkle were the strongest facial factors explaining $21 \%$ and $13 \%$ of the influence of aversion to ambiguity on $\mathrm{TI}$.

\section{What is the relevance of our findings for clinical practice?}

$\mathrm{TI}$ is a common phenomenon affecting $50 \%$ to $90 \%$ of physicians who manage patients with chronic medical conditions (e.g. hypertension, diabetes, chronic obstructive pulmonary disease, multiple sclerosis, among others).(15, 41-44). TI has been associated with poorer patient outcomes and higher health care costs due to 
the lack of appropriate treatment escalation (affecting one out of six clinical encounters) leading to higher hospitalizations, greater disability, and lower productivity.(14, 18, 24, 45) It may occur with insufficient knowledge integration and knowledge-to-action gaps as a result of automatic responses leading to suboptimal therapeutic decisions. Specifically, neurologists caring for MS patients sometimes fail to integrate presented information (e.g. MS severity, relapses within the last three years, imaging findings with the risk of disease progression) with best practice recommendations.(46-49) In the present study, we found an association between facial and emotional expressions with aversion to ambiguity and TI.

Prior studies have demonstrated that interventions increasing physicians' arousal or awareness (e.g. through warning and categorization strategies) were associated with more accurate diagnostic or therapeutic decisions. $(50,51)$ A recent randomized clinical trial showed that neurologists who received the traffic light system educational intervention had a 70\% reduction in TI (manuscript submitted for publication in Feb 2019). Furthermore, several studies demonstrated a link between specific emotions (e.g. disgust) increasing attention at early stages of visual processing.(52) These prior findings, together with observations from the current study suggest that emotional expressions and strategies that enhance participants awareness (via increasing attention or arousal) may reduce $\mathrm{TI}$.

Furthermore, our findings of (i) brow furrow being associated with both increased $\mathrm{TI}$ and ambiguity aversion and (ii) disgust being associated with both reduced $\mathrm{TI}$ and lower ambiguity aversion indicates that common emotional factors may contribute to both behaviors. In our previous studies, aversion to ambiguity was the most significant physician-level factor associated with TI.(16, 18, 53) Given the limited training in risk management and formal learning in medical decisions, physicians are clearly vulnerable when handling decisions under uncertainty, especially when having aversion to ambiguity.(54-56) Taken together, these findings suggest that interventions reducing TI may partly rely on emotional factors (51) and that emotional factors may play a more important role for medical decision making than hitherto assumed. 
What brain pathways that may underpin the link between emotions and TI?

Previous studies suggest that the neural mechanisms mediating the relation between affect and decisions depend on a participant's emotional arousal and engagement with the specific choice to be made. (3) For example, disgust has been associated with the activation of the insular cortex which may lead to increased arousal modulating the neural responses to aversion to ambiguity, which results in influencing subsequent decision-making.(57, 58) Disgust was also shown to increase arousal by modulating emotion-specific attention.(52) This finding is also consistent with an increased arousal score associated with disgust (and its muscle components) in our study.

Traditionally, the striatum, the amygdala, the medial prefrontal, orbitofrontal and insular cortices are thought to process emotional aspects of the decision-making process. $(3,7)$ Moreover, the dorsolateral and anterior prefrontal cortices and the posterior parietal cortex may modulate cognitive aspects of decisions.(4) Previous studies showed that stress reduces activity in dorsolateral and orbital parts of prefrontal cortex while it enhances amygdala activity, leading to decreased goal-directed behavior and increased emotional responses (e.g. fear, disgust, contempt).(59,60) Findings from our study are in keeping with this proposed framework as they support an association between facial metrics and emotional expressions (disgust) which may increase participant's awareness/arousal (reflected by increased arousal scores) regarding a compelling decision, thereby showing a reduction in ambiguity aversion and lowering the likelihood of TI (Figure 5). We could speculate that muscle activations associated with disgust and surprise may reflect increased activity of the insular cortex and amygdala leading to greater arousal and lower TI. Further studies using time-resolved neural methods are needed to test this hypothesis.

\section{Limitations}

Our study has a number of significant limitations. First, although we used a validated software to detect facial muscle activation, the association with emotional expressions may require further assessment. Second, there is 
a high variability of emotional expressions when participants are exposed to a specific stimulus. As such, our results should be interpreted with caution considering there are no other similar studies available for comparison. Third, the sample size is small affecting the precision of our results (e.g. wider confidence intervals). Fourth, the prevalence of emotional expressions was relatively low likely due to: i) the strict pre-specified correlation mapping used by AFFDEX software (that combines the concomitant activation of several facial muscles to code for a single emotion)(19), and ii) emotions are inherently social, and therefore more neutral expressions are commonly observed when participants are exposed to computer-based simulated scenarios.(61, 62) Finally, our stimulus was based on case-scenarios that may not truly reflect the therapeutic decisions in clinical practice.

Despite these limitations, our study is the first to show that facial muscle activations and emotional expressions are associated with therapeutic decisions made by physicians who care for MS patients.

\section{Conclusions}

This information helps improve our understanding of the influence of emotional expressions on physicians' therapeutic decisions. These findings, in conjunction with results from a prior study that demonstrated the benefits of an educational intervention on reducing TI have practical clinical implications. With further studies, it may be possible to identify physicians at high risk of having TI by evaluating physical, emotional, and behavioral responses (aversion to ambiguity) and tailor educational interventions to these individuals. Identifying and administering appropriate educational interventions in such situations may facilitate optimal therapeutic decisions in chronic diseases, resulting in better patient outcomes and lower health care costs. 


\section{Figure legends:}

\section{Figure 1: Study design and facial landmarks}

(A) Sequence of study events. After answering demographic and practice-based questions and determining medical and financial ambiguity aversion, participants listened to a case-scenario and then viewed 6 therapeutic choices. This procedure was repeated for each of the 10 case-scenarios ranging from 25 to 50 seconds. All the stimuli remained on the screen until the participant selected one of the therapeutic choices. Then, the participant was able to see the next screen and play the next case-scenario. The dots between the screen presenting the therapeutic choices \#1 and the case-scenario \#10 represent the progression through scenarios \#2-\#9. (B) Facial landmarks. The region of interest in AFFDEX software contains the whole face including eyes, mouth and nose. Each of the 34 facial landmarks are the main unit of study to represent 20 facial expression metrics that are mapped to represent emotional expressions (https://developer.affectiva.com/mappingexpressions-to-emotions/). This figure illustrates the representation of the data at a particular time-point of the study, including: facial metrics, screens of the stimulus presentation, and time landmarks according to the study design and flow. A value of zero indicates no evidence and a value of one the highest evidence that a certain facial metric or emotion is fully expressed. (23)

\section{Figure 2. Facial muscle activations overall and in relation to TI status.}

(A) Overall proportion of facial muscle activations in ascending order. Values at the top of the bars represent the proportion of muscle activation during the study period. (B) Represents the distribution of facial muscle activations as shown in (A) stratified by responses with (red) and without (blue) TI. Values within bars represent the proportion of muscle activations by TI status. For example, brow furrow activation was observed in $19.1 \%$ of responses, $14.7 \%$ among participants with $\mathrm{TI}$ and the remaining $4.4 \%$ among participants without $\mathrm{TI}$. The $\mathrm{x}$-axis represents individual facial metric as identified by the AFFDEX software for activations greater than $1 \%$. (C) Overall proportion of emotional expressions in ascending order. Values at the top of the bars represent the proportion of emotional expressions during the study period. (D) Represents the distribution of emotional expressions as shown in (C) stratified by responses with (red) and without (blue) TI. For example, disgust was observed in $8 \%$ of responses, $5.8 \%$ among participants without $\mathrm{TI}$ and the remaining $2.2 \%$ among participants with TI. The $\mathrm{x}$-axes represent each individual emotional expression as mapped by the AFFDEX software.

* indicates $p$-values $<0.01,{ }^{* *}$ indicates $p$-values $<0.001,+$ indicates $p$-values $<0.05->0.01$ for differences between participants with and without TI. 


\section{Figure 3. Predicted TI score as a function of degrees of aversion to ambiguity}

The mixed linear regression models were adjusted for age, sex, participants' expertise (MS expert vs. general neurologist), disgust (panel A) or facial muscle activation (brow furrow, mouth opening, and lip suck) (Panel B). The gray are represents the $95 \% \mathrm{Cl}$ of the predicted $\mathrm{TI}$ score.

For the model accounting for emotional expression (disgust) (Panel A), the R-squared of 0.35 represents the proportion of the variability of the TI score explained by the model. For every $20 \%$ increase in the degree of aversion to ambiguity (e.g. from $10 \%$ to $30 \%$ or from $50 \%$ to $90 \%$ ), there was a $21.5 \%$ increment in the $\mathrm{TI}$ score. For the model accounting for facial muscle activations (Panel B), the R-squared of 0.38 represents the proportion of the variability of the TI score explained by the model. For every $20 \%$ increase in the degree of aversion to ambiguity (e.g. from $10 \%$ to $30 \%$ or from $50 \%$ to $90 \%$ ), there was a $19.5 \%$ increment in the $\mathrm{TI}$ score. The gray are represents the $95 \% \mathrm{Cl}$ of the predicted $\mathrm{TI}$ score.

Figure 4. Mediation analysis: graphs derived from structural equation models with a single mediator (see also explanatory figure $\mathrm{e} 1$ in the appendix)

(A) Structural equation model graph for the modulation of brow furrow (mediator) on the relationship between aversion to ambiguity (independent variable) and therapeutic inertia score (outcome). The R-squared for the model was 0.38. (B) Structural equation model graph for the modulation of nose wrinkle (mediator) on the relationship between aversion to ambiguity (independent variable) and therapeutic inertia score (outcome). The R-squared for the model was 0.34. (C) Structural equation model graph for the modulation of disgust (mediator) on the relationship between aversion to ambiguity (independent variable) and therapeutic inertia score (outcome). The R-squared for the model was $0.35 \mathrm{Age}$, sex and MS expertise were included as covariates. Values next to the arrows represent $\beta$ coefficients, $\varepsilon$ represent the variance of the mediator and outcome of interest (TI score). Values within each square box represent the mean (upper values) and variance (lower value) of each variable included in the models.

* represents a $p$-value $<0.001,+$ represents a $p$-value $<0.05$ and $>0.01$ for the total effect models.

\section{Figure 5. Schematic representation of factors associated with therapeutic inertia (TI)}

There is a direct effect of ambiguity aversion and $\mathrm{Tl}$ and an indirect effect of facial muscle activations (e.g. brow furrow, lip suck) and emotional expressions (e.g. disgust) modulating the relationship between ambiguity aversion and therapeutic inertia. Demographic and practice factors (e.g. number of MS patients seen per week) may also contribute to $\mathrm{TI}$. 
Table 1. Baseline characteristics of participants

\begin{tabular}{|c|c|}
\hline Characteristics & $\begin{array}{c}\text { Total (\%) } \\
n=34\end{array}$ \\
\hline Age (mean $\pm S D$ ), in years & $44.6 \pm 11.6$ \\
\hline Age $\geq 50$ years & $13(38.2)$ \\
\hline \multicolumn{2}{|l|}{ Sex } \\
\hline Female & $13(38.2)$ \\
\hline \multicolumn{2}{|l|}{ Specialty } \\
\hline MS specialists & $20(58.8)$ \\
\hline General Neurologists who care for MS patients & $14(41.2)$ \\
\hline \multicolumn{2}{|l|}{ Practice Setting } \\
\hline Academic & $28(82.4)$ \\
\hline Community & $6(17.6)$ \\
\hline \multicolumn{2}{|l|}{$\%$ time in clinical practice } \\
\hline $50-74 \%$ of their time & $16(47.2)$ \\
\hline Greater than $75 \%$ & $15(44.1)$ \\
\hline Years in practice (mean \pm SD) & $12.5 \pm 11.8$ \\
\hline MS patients seen per week (mean \pm SD) & $23.1 \pm 15.8$ \\
\hline Author of a peer-reviewed publication in the last 12 months & $22(64.7)$ \\
\hline
\end{tabular}

Numbers in brackets indicate percentages 
Table 2. Relationship between aversion to ambiguity and therapeutic inertia

\begin{tabular}{|l|c|c|c|c|}
\hline & $\begin{array}{c}\text { N of individual } \\
\text { responses }\end{array}$ & TI score * & $\begin{array}{c}\text { TI t } \\
\text { (present/absent) }\end{array}$ & $\begin{array}{c}\text { Area } \\
\text { under the } \\
\text { curve }\end{array}$ \\
\hline & $\begin{array}{c}\text { n individual responses } \\
\text { among participants } \\
\text { with aversion to } \\
\text { ambiguity /total } \\
\text { number of responses } \\
(\%)\end{array}$ & $\begin{array}{c}\text { (\% Coefficient } \\
(95 \% \mathrm{Cl})\end{array}$ & OR (95\%Cl) & \\
\hline $\begin{array}{l}\text { Aversion to Ambiguity } \\
\text { (Financial domain) }\end{array}$ & $380 / 680(55.9)$ & $0.21(0.16-0.26)$ & $1.56(1.32-1.86)$ & 0.82 \\
\hline $\begin{array}{l}\text { Aversion to Ambiguity } \\
\text { (Health domain) }\end{array}$ & $460 / 680(66.7)$ & $0.06(0.03-0.08)$ & $1.12(1.02-1.22)$ & 0.78 \\
\hline
\end{tabular}

* Mixed linear regression models for TI score (dependent variable) adjusted for age, sex, MS expert and aversion to ambiguity (independent variables)

† Mixed logistic regression models for TI (dependent variable) adjusted for age, sex, MS expert and aversion to ambiguity (independent variables). 
Figure 1. Study flow (A) and facial landmarks (B)

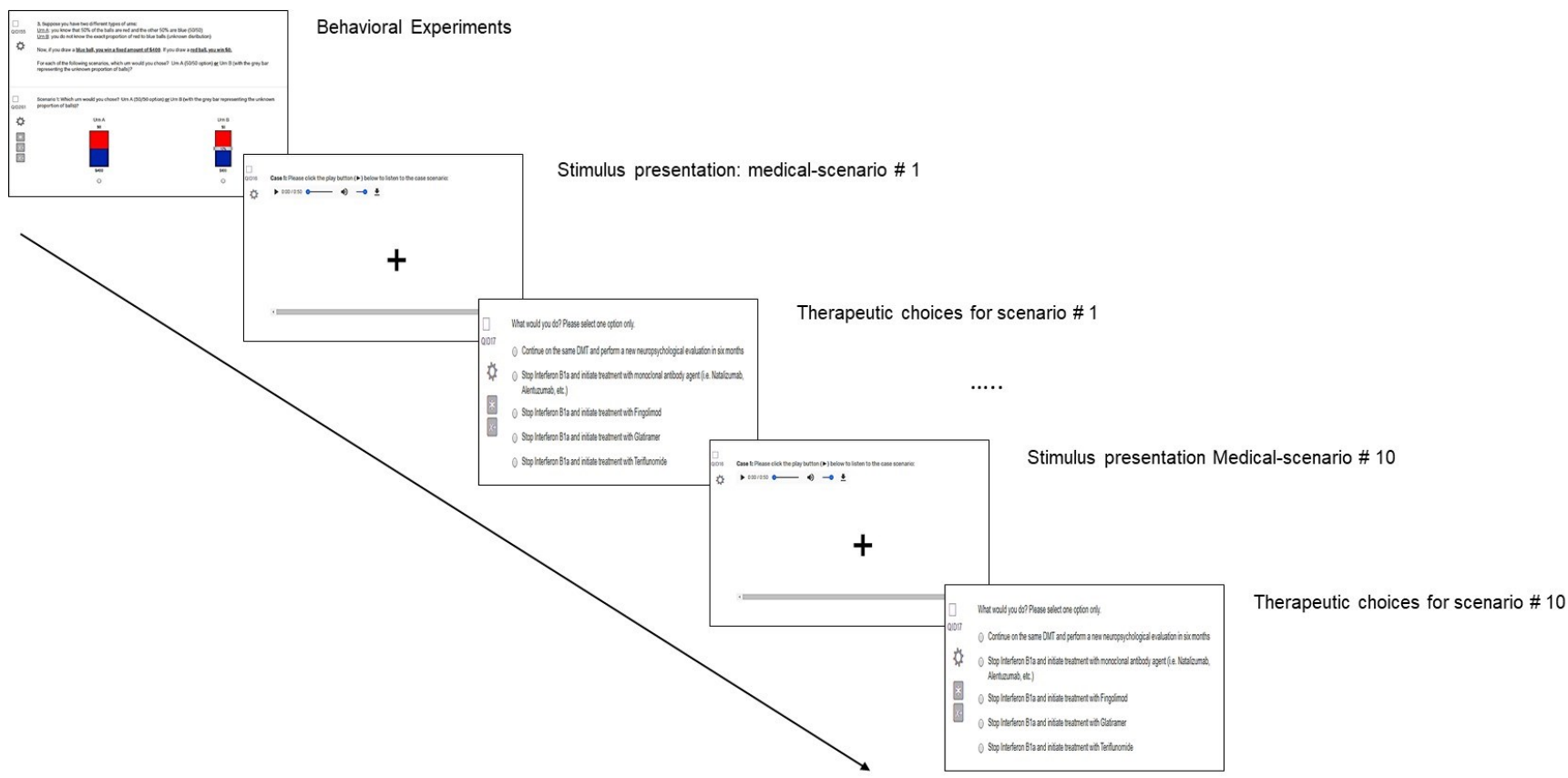

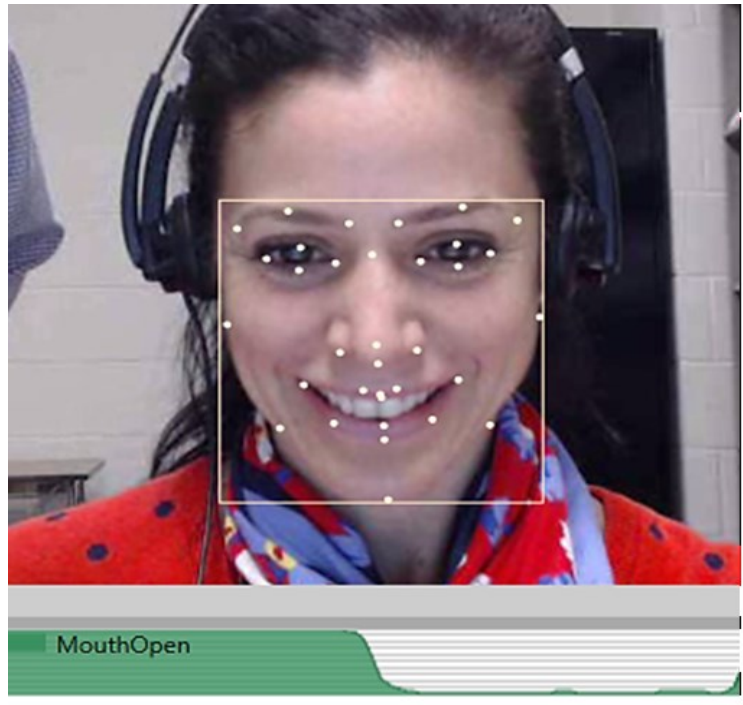

Lip Stretch

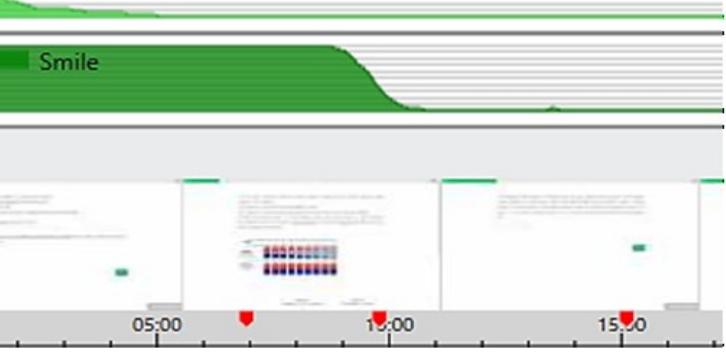

Facial landmarks

Facial muscle activity

Stimulus presentation

Time period 


\section{Figure 2. (A) Prevalence of facial muscle activations}

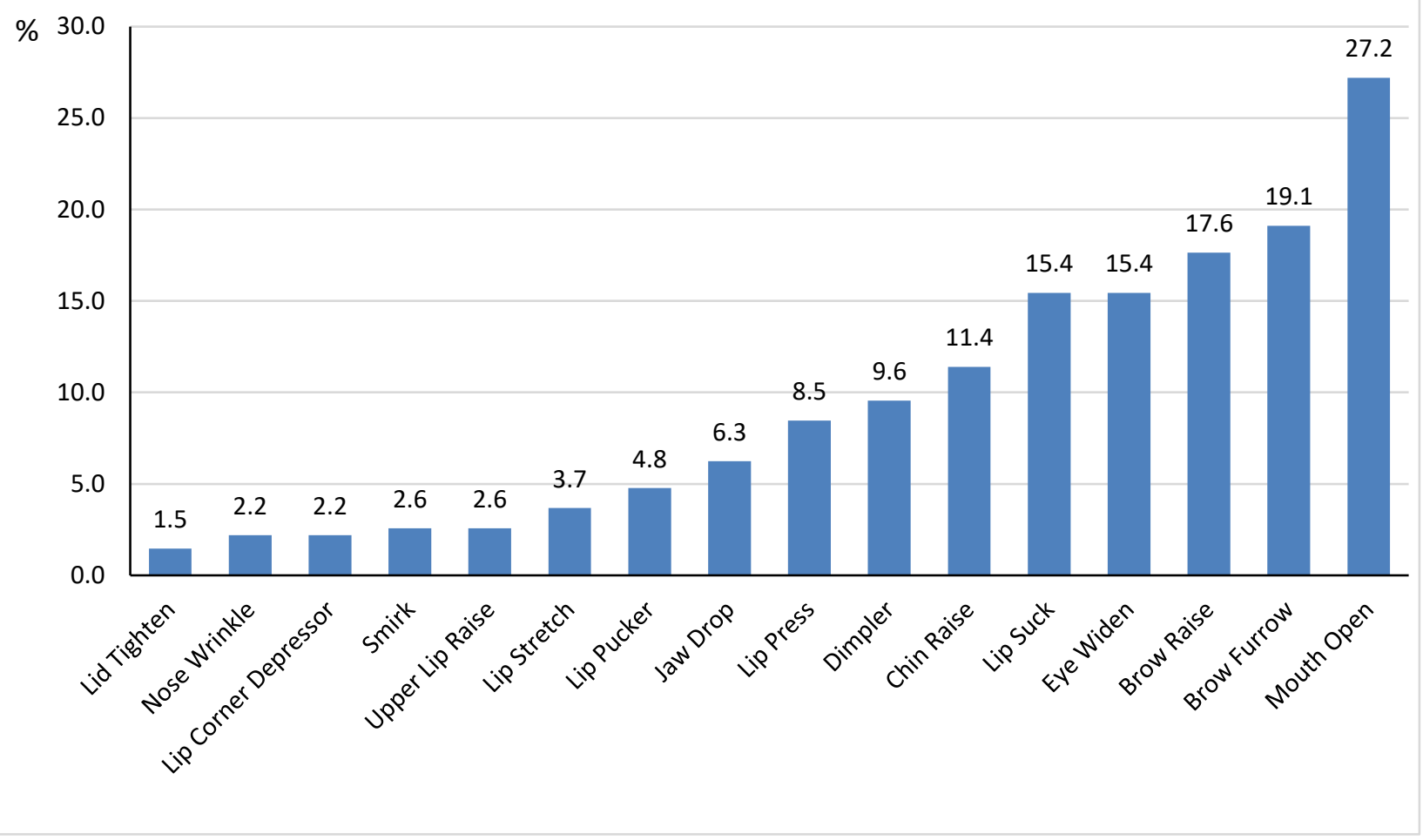

Figure 2. (B) Prevalence of facial muscle activations by TI status

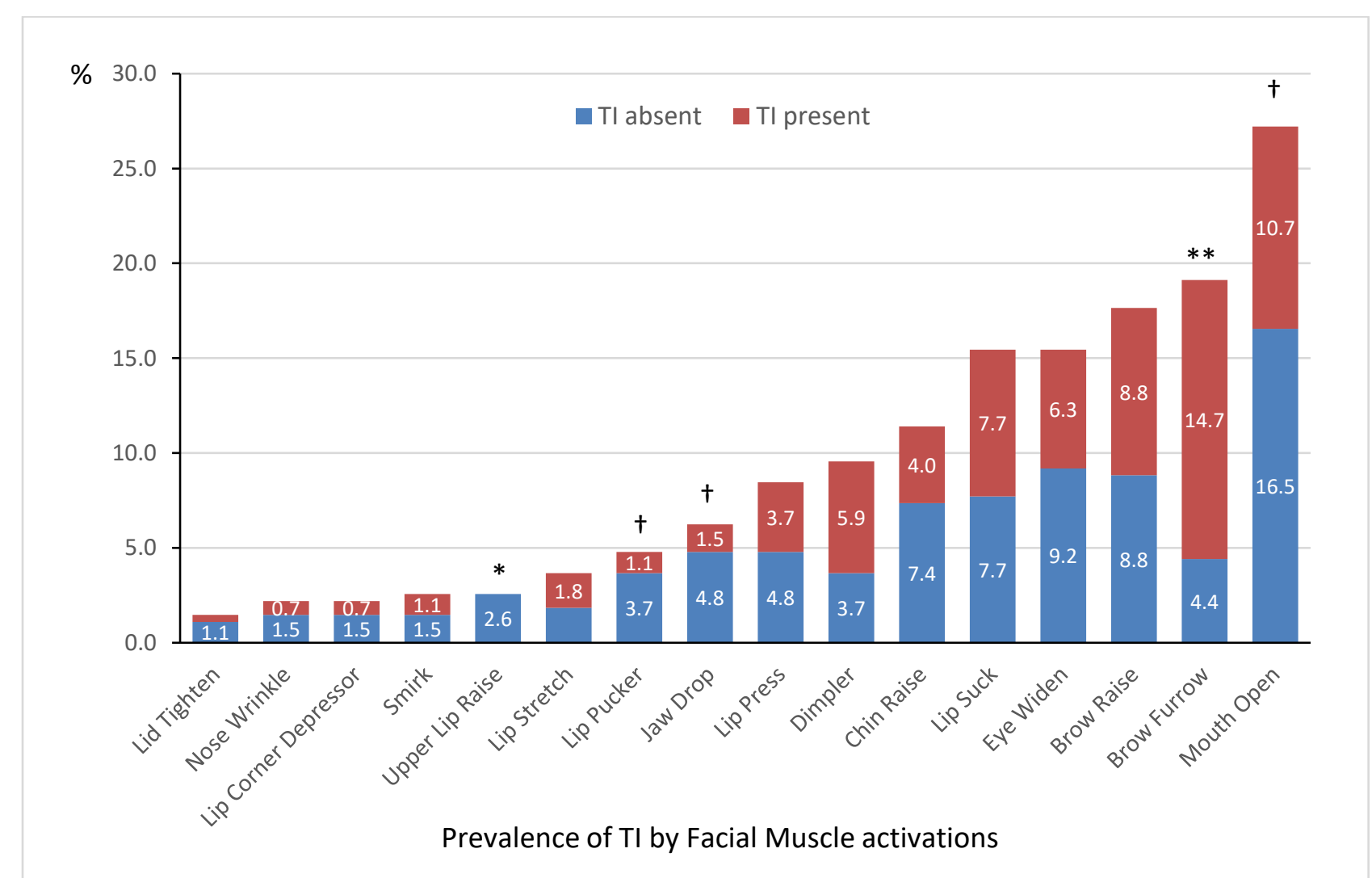




\section{(C) Prevalence of emotional expressions overall}

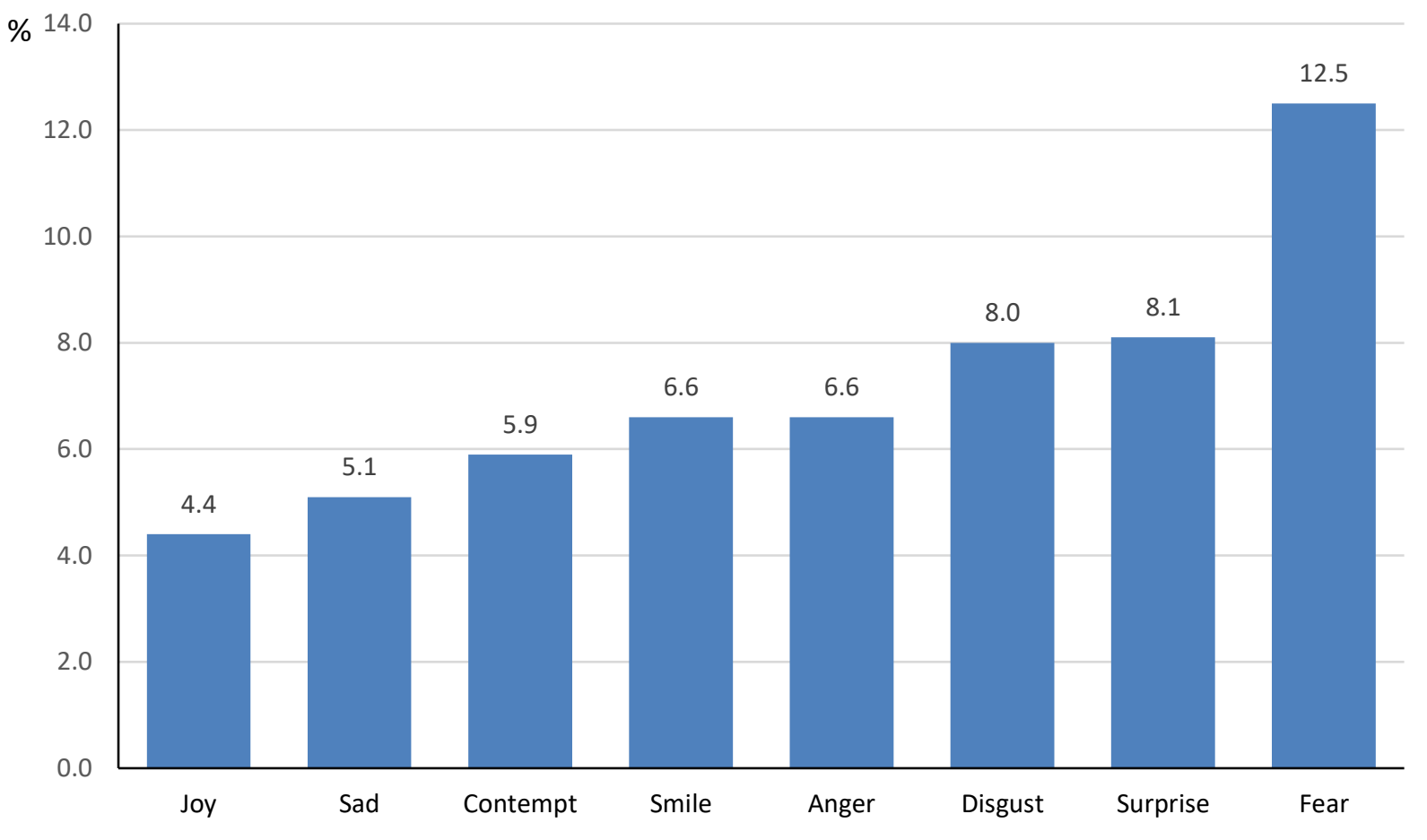

\section{(D) Prevalence of emotional expressions by TI status}

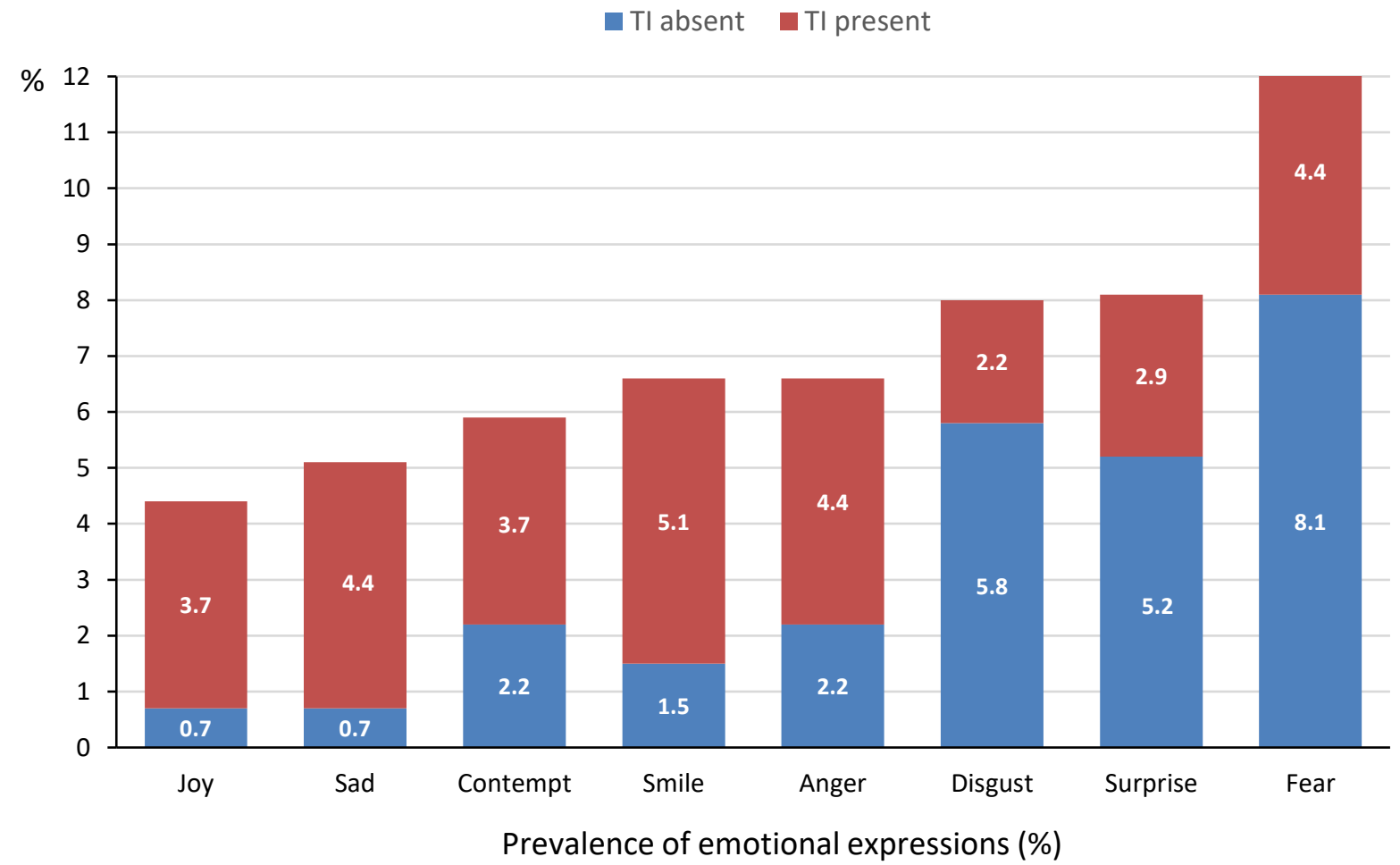


Figure 3. Predicted TI score by degrees of aversion to ambiguity score

\section{Panel A: Accounting for the effect of emotional expression (disgust)}

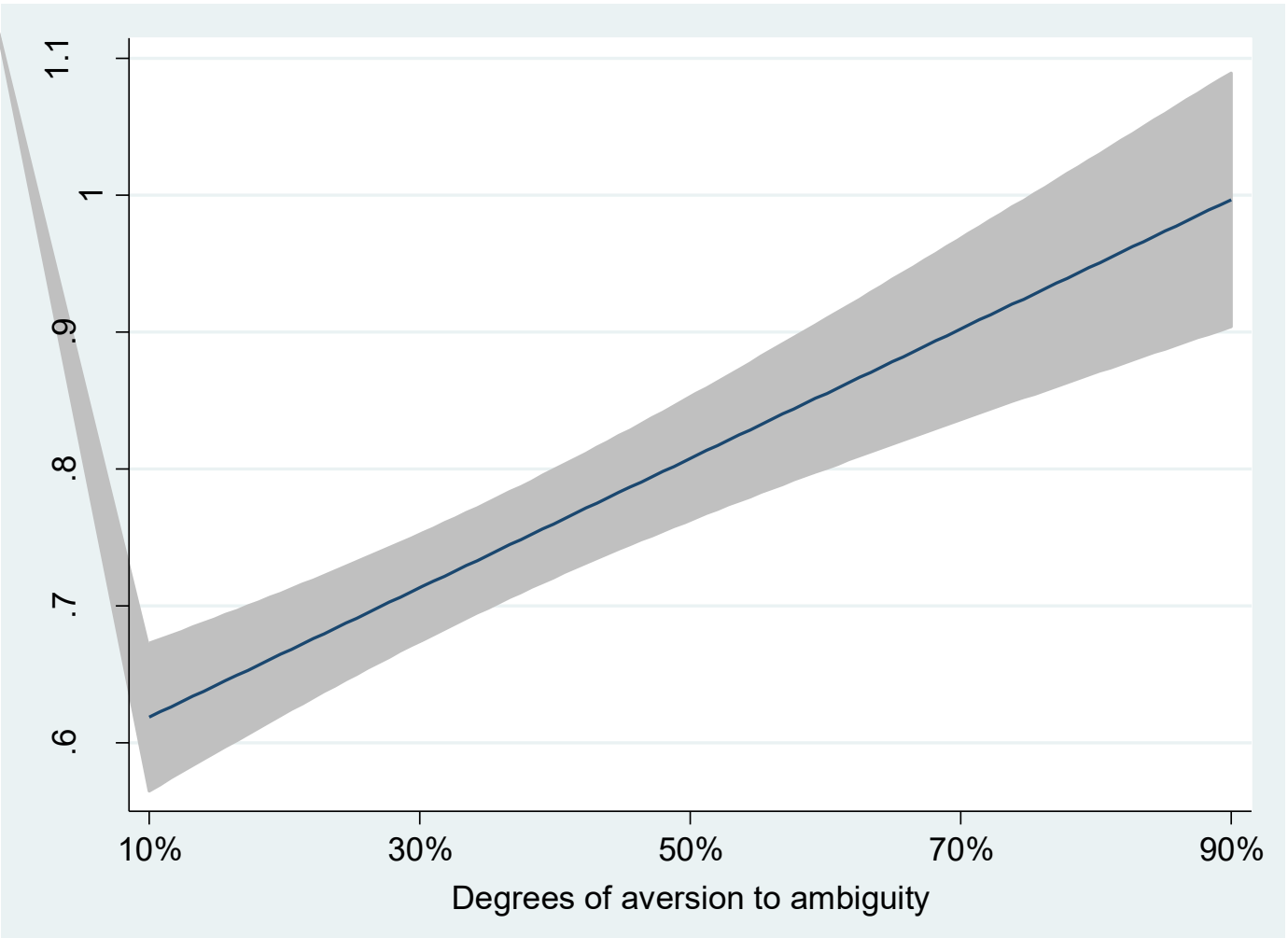

Panel B: accounting for the effect of muscle activations

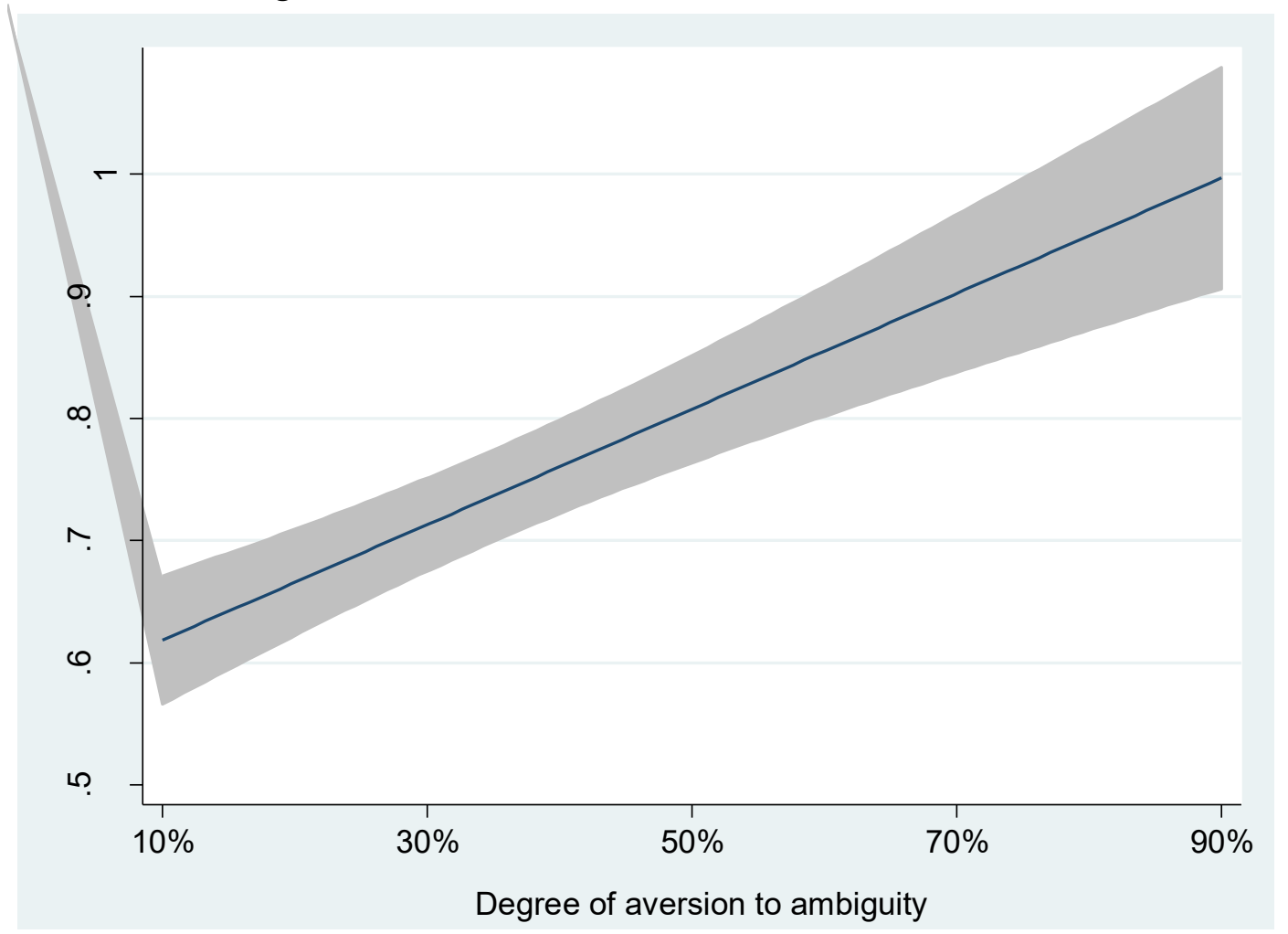


Figure 4. Graphs derived from the structural equation modelling representing the mediation analysis (see also explanatory figure in the appendix)

Panel A. Direct, indirect, and total effect of aversion to ambiguity on TI with brow furrow as mediator

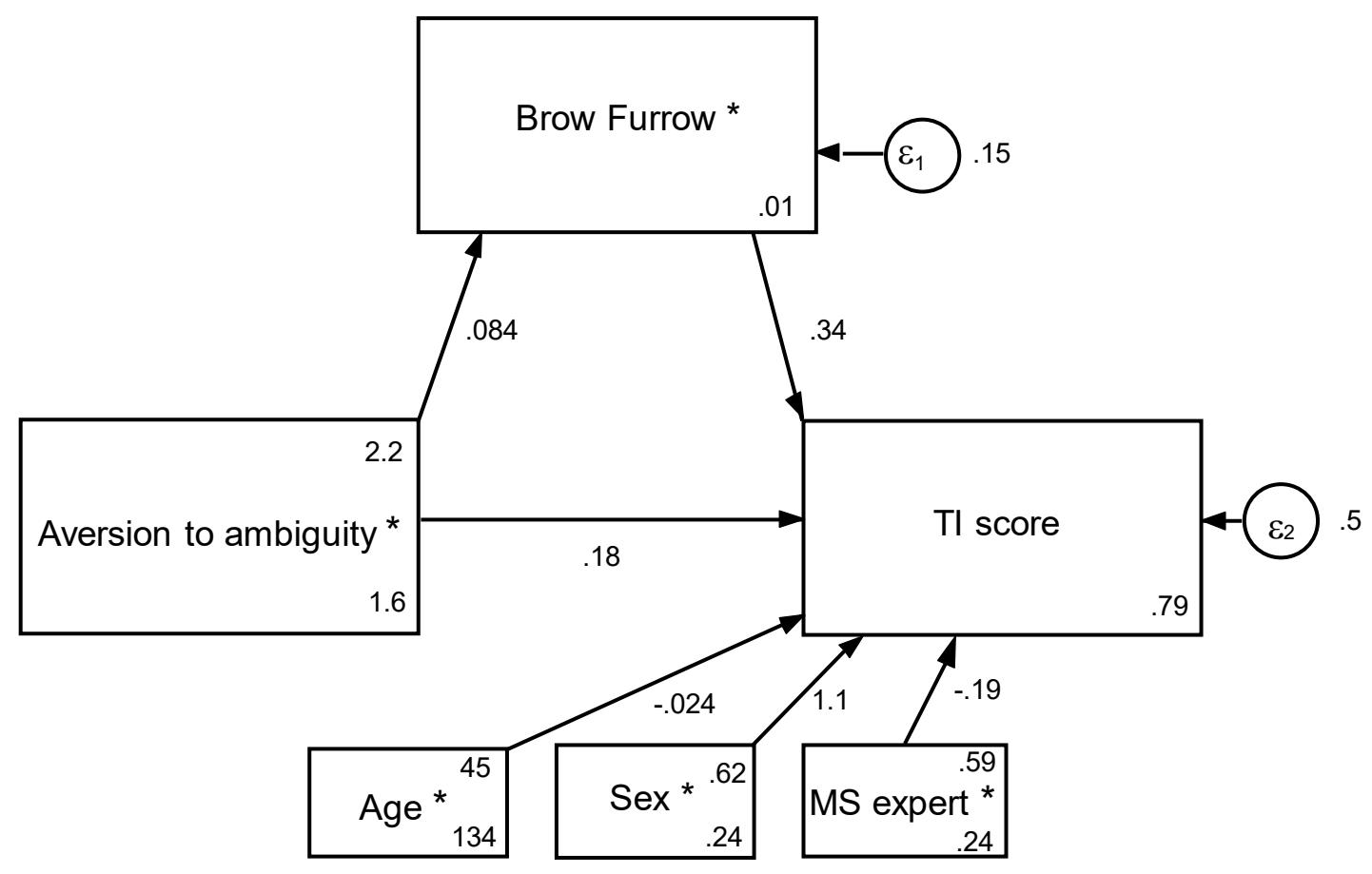

Panel B. Direct, indirect, and total effect of aversion to ambiguity on TI with nose wrinkle as mediator

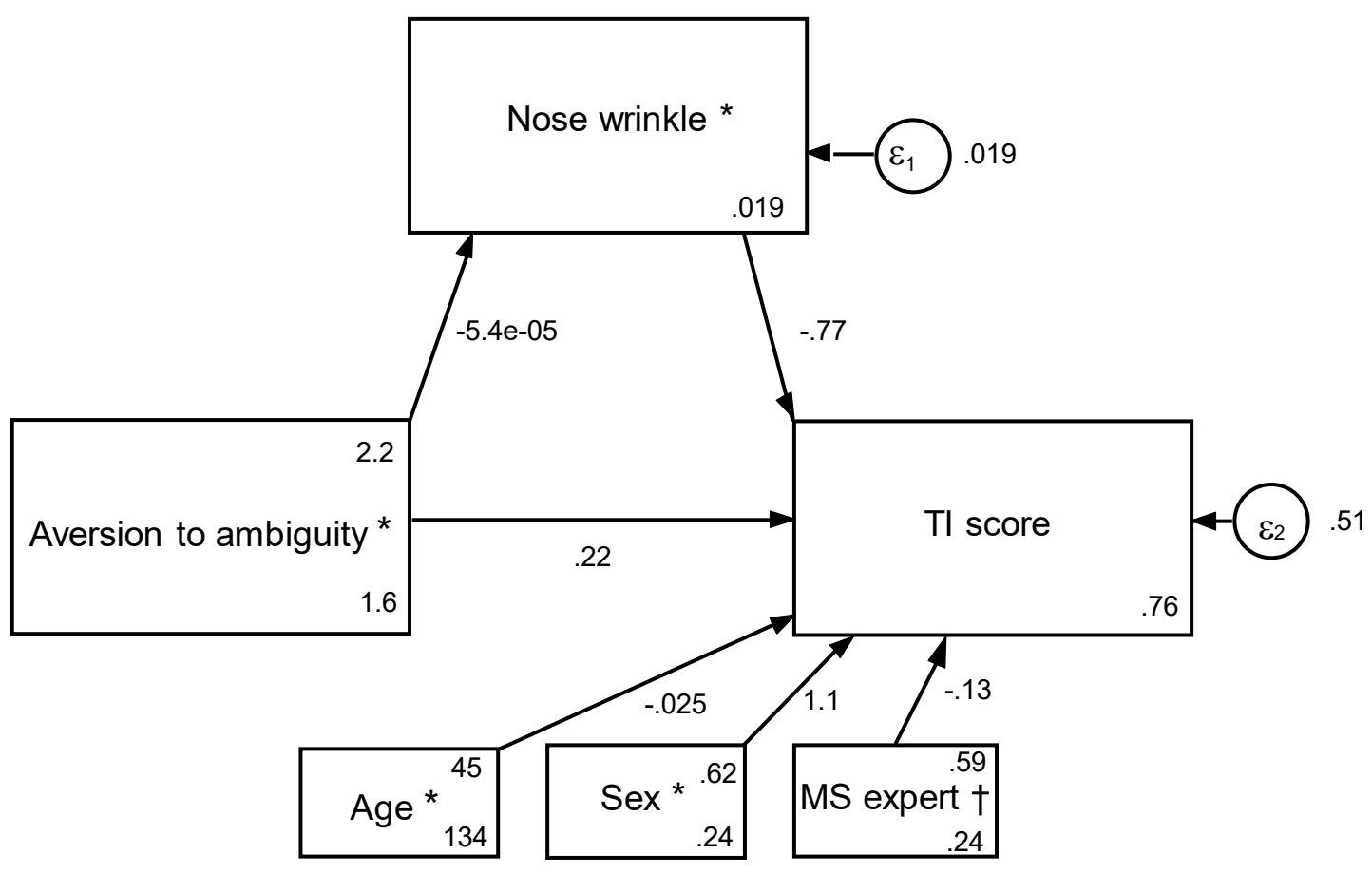


Panel C. Direct, indirect, and total effect of aversion to ambiguity on $\mathrm{TI}$ with disgust as mediator

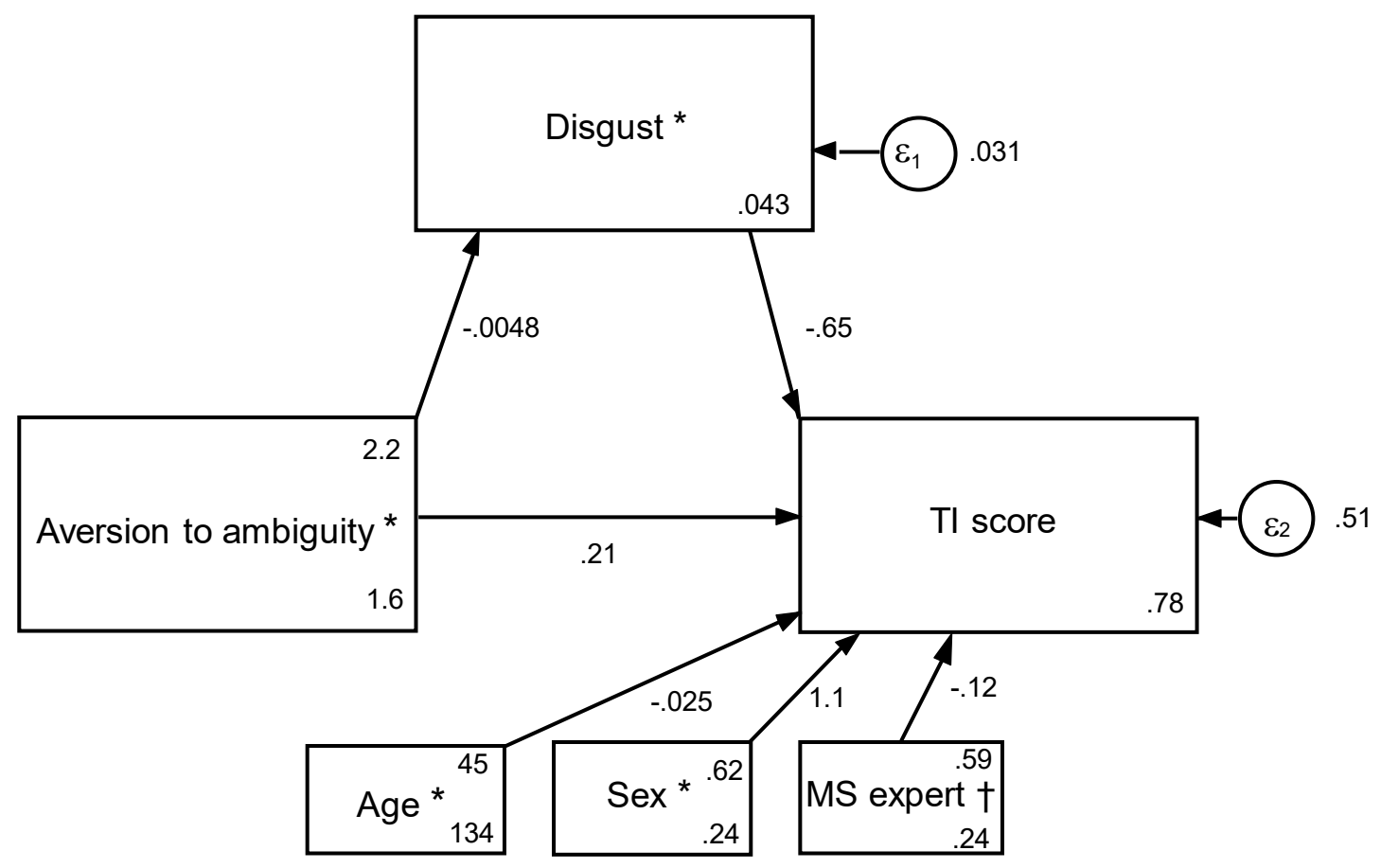


Figure 5. Proposed pathways associated with Therapeutic Inertia: integration of demographic, physical, emotional, and behavioral mechanisms

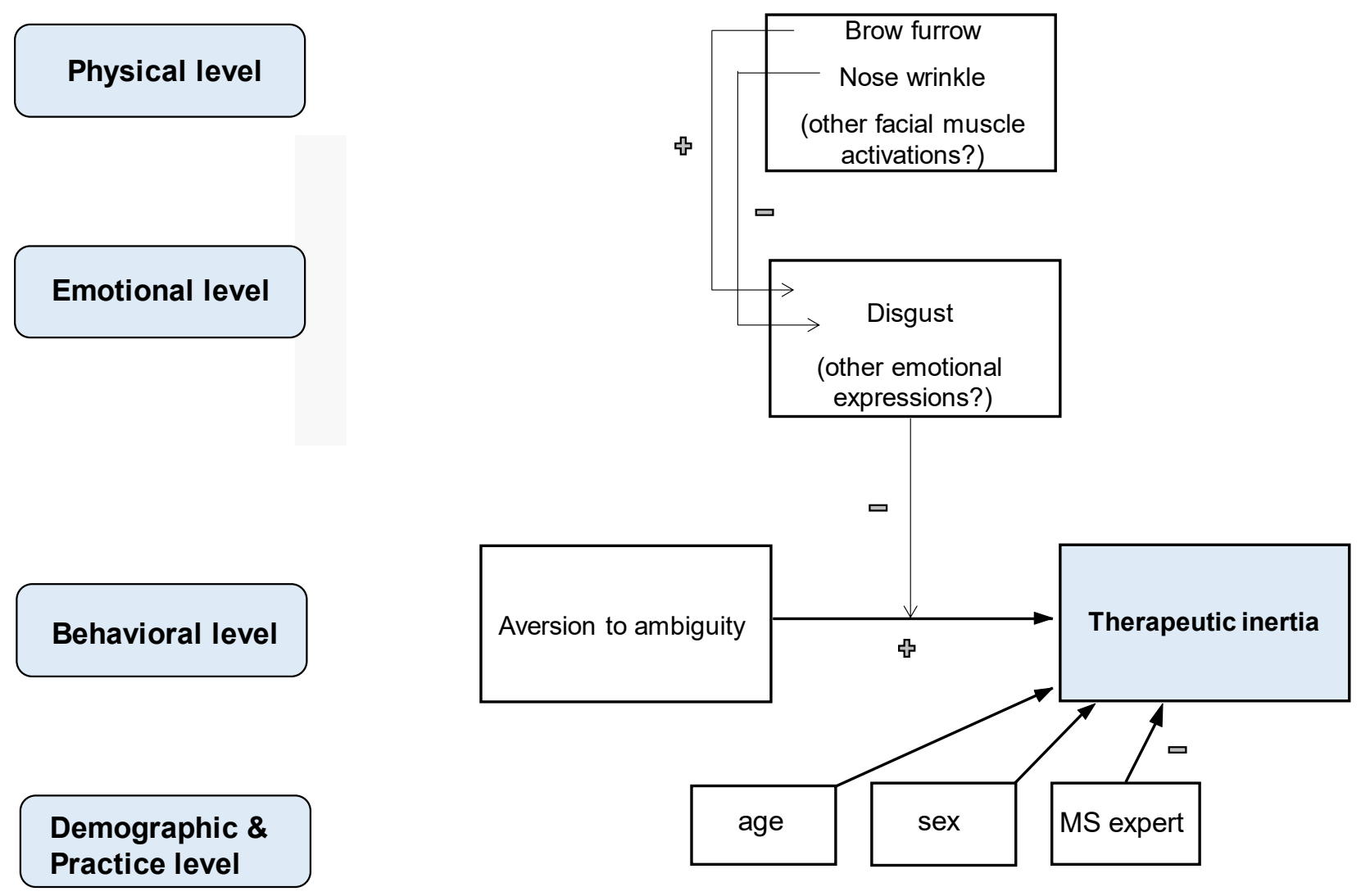




\section{References}

1. Ekman P. Emotions revealed: Recognizing faces and feelings to improve communication and emotional life: Macmillan; 2007.

2. d'Acremont M, Bossaerts P. Decision making: how the brain weighs the evidence. Curr Biol. 2012;22(18):R808-10.

3. Phelps EA, Lempert KM, Sokol-Hessner P. Emotion and Decision Making: Multiple Modulatory Neural Circuits. Annual Review of Neuroscience. 2014;37(1):263-87.

4. Cohen JD. The Vulcanization of the Human Brain: A Neural Perspective on Interactions Between Cognition and Emotion. Journal of Economic Perspectives. 2005;19(4):3-24.

5. Ekman P, Friesen WV. Unmasking the face: A guide to recognizing emotions from facial clues: Ishk; 2003.

6. Lerner JS, Gonzalez RM, Small DA, Fischhoff B. Effects of Fear and Anger on Perceived Risks of Terrorism:A National Field Experiment. Psychological Science. 2003;14(2):144-50.

7. Lerner JS, Li Y, Valdesolo P, Kassam KS. Emotion and Decision Making. Annual Review of Psychology. 2015;66(1):799-823.

8. Kligyte V, Connelly S, Thiel C, Devenport L. The Influence of Anger, Fear, and Emotion Regulation on Ethical Decision Making. Human Performance. 2013;26(4):297-326.

9. Chesney SA, Reiter K. Thinking positively: Optimism and emotion regulation predict interpretation of ambiguous information AU - Gordon, Nakia S. Cogent Psychology. 2016;3(1):1195068.

10. FeldmanHall O, Glimcher P, Baker AL, Phelps EA. Emotion and decision-making under uncertainty: Physiological arousal predicts increased gambling during ambiguity but not risk. Journal of experimental psychology General. 2016;145(10):1255-62.

11. O'Connor PJ, Sperl-Hillen JAM, Johnson PE, Rush WA, Biltz G. Clinical Inertia and Outpatient Medical Errors. In: Henriksen K, Battles JB, Marks ES, Lewin DI, editors. Advances in Patient Safety: From Research to Implementation (Volume 2: Concepts and Methodology). Advances in Patient Safety. Rockville (MD)2005.

12. Mohan AV, Phillips LS. Clinical inertia and uncertainty in medicine. JAMA. 2011;306(4):383; author reply -4 .

13. Okonofua EC, Simpson KN, Jesri A, Rehman SU, Durkalski VL, Egan BM. Therapeutic inertia is an impediment to achieving the Healthy People 2010 blood pressure control goals. Hypertension. 2006;47(3):345-51.

14. Burks J, Marshall TS, Ye X. Adherence to disease-modifying therapies and its impact on relapse, health resource utilization, and costs among patients with multiple sclerosis. ClinicoEconomics and outcomes research : CEOR. 2017;9:251-60.

15. Cooke CE, Sidel M, Belletti DA, Fuhlbrigge AL. Review: clinical inertia in the management of chronic obstructive pulmonary disease. COPD. 2012;9(1):73-80.

16. Saposnik G, Montalban X. Therapeutic Inertia in the New Landscape of Multiple Sclerosis Care. Front Neurol. 2018;9:174.

17. Lebeau JP, Cadwallader JS, Aubin-Auger I, Mercier A, Pasquet T, Rusch E, et al. The concept and definition of therapeutic inertia in hypertension in primary care: a qualitative systematic review. BMC Fam Pract. 2014;15:130.

18. Saposnik G, Sempere AP, Prefasi D, Selchen D, Ruff CC, Maurino J, et al. Decision-making in Multiple Sclerosis: The Role of Aversion to Ambiguity for Therapeutic Inertia among Neurologists (DIScUTIR MS). Front Neurol. 2017;8:65.

19. Stöckli S, Schulte-Mecklenbeck M, Borer S, Samson AC. Facial expression analysis with AFFDEX and FACET: A validation study. Behavior Research Methods. 2018;50(4):1446-60.

20. McDuff D, Kodra E, Kaliouby RE, LaFrance M. A large-scale analysis of sex differences in facial expressions. PLoS One. 2017;12(4):e0173942. 
21. Saposnik G, Sempere AP, Raptis R, Prefasi D, Selchen D, Maurino J. Decision making under uncertainty, therapeutic inertia, and physicians' risk preferences in the management of multiple sclerosis (DIScUTIR MS). BMC Neurol. 2016;16(1):58.

22. Anderson LR, Mellor JM. Predicting health behaviors with an experimental measure of risk preference. J Health Econ. 2008;27(5):1260-74.

23. Levy I, Snell J, Nelson AJ, Rustichini A, Glimcher PW. Neural representation of subjective value under risk and ambiguity. Journal of neurophysiology. 2010;103(2):1036-47.

24. Saposnik G, Sempere AP, Raptis R, Prefasi D, Selchen D, Maurino J. Decision making under uncertainty, therapeutic inertia, and physicians' risk preferences in the management of multiple sclerosis (DIScUTIR MS). BMC Neurol. 2016;16:58.

25. Saposnik G, Montalban X, Selchen D, Terzaghi MA, Bakdache F, Montoya A, et al. Therapeutic Inertia in Multiple Sclerosis Care: A Study of Canadian Neurologists. Front Neurol. 2018;9:781.

26. iMotions. Facial Expression Analysis. Boston, MA 02111; 2016.

27. Freedman MS, Selchen D, Prat A, Giacomini PS. Managing Multiple Sclerosis: Treatment Initiation, Modification, and Sequencing. Canadian Journal of Neurological Sciences / Journal Canadien des Sciences Neurologiques. 2018:1-15.

28. Freedman MS, Selchen D, Arnold DL, Prat A, Banwell B, Yeung M, et al. Treatment optimization in MS: Canadian MS Working Group updated recommendations. Can J Neurol Sci. 2013;40(3):307-23.

29. Noyes K, Weinstock-Guttman B. Impact of diagnosis and early treatment on the course of multiple sclerosis. Am J Manag Care. 2013;19(17 Suppl):s321-31.

30. Sormani MP, Rio J, Tintore M, Signori A, Li D, Cornelisse P, et al. Scoring treatment response in patients with relapsing multiple sclerosis. Mult Scler. 2013;19(5):605-12.

31. Duquette P, Giacomini PS, Bhan V, Hohol M, Schecter R. Balancing Early Aggression Against Risk of Progression in Multiple Sclerosis. Can J Neurol Sci. 2016;43(1):33-43.

32. Prosperini L, Gianni C, Leonardi L, De Giglio L, Borriello G, Galgani S, et al. Escalation to natalizumab or switching among immunomodulators in relapsing multiple sclerosis. Mult Scler. 2012;18(1):64-71.

33. Harding K, Williams O, Willis M, Hrastelj J, Rimmer A, Joseph F, et al. Clinical Outcomes of Escalation vs Early Intensive Disease-Modifying Therapy in Patients With Multiple Sclerosis. JAMA Neurol. 2019.

34. Prosperini L, Mancinelli CR, De Giglio L, De Angelis F, Barletta V, Pozzilli C. Interferon beta failure predicted by EMA criteria or isolated MRI activity in multiple sclerosis. Mult Scler. 2014;20(5):566-76.

35. Bermel RA, You X, Foulds P, Hyde R, Simon JH, Fisher E, et al. Predictors of long-term outcome in multiple sclerosis patients treated with interferon beta. Ann Neurol. 2013;73(1):95-103.

36. MacKinnon DP, Fairchild AJ, Fritz MS. Mediation Analysis. Annual Review of Psychology. 2007;58(1):593-614.

37. VanderWeele TJ. Mediation Analysis: A Practitioner's Guide. Annual Review of Public Health. 2016;37(1):17-32.

38. Valeri L, Vanderweele TJ. Mediation analysis allowing for exposure-mediator interactions and causal interpretation: theoretical assumptions and implementation with SAS and SPSS macros. Psychol Methods. 2013;18(2):137-50.

39. Fairchild AJ, MacKinnon DP. A general model for testing mediation and moderation effects. Prev Sci. 2009;10(2):87-99.

40. Verkuilen J. Explanatory Item Response Models: A Generalized Linear and Nonlinear Approach by P. de Boeck and M. Wilson and Generalized Latent Variable Modeling: Multilevel, Longitudinal and Structural Equation Models by A. Skrondal and S. Rabe-Hesketh. Psychometrika. 2006;71(2):415-8.

41. Blasco M, Perez-Martinez P, Lahoz C. Decalogue of the Spanish Society of Arteriosclerosis to reduce therapeutic inertia. Clin Investig Arterioscler. 2017;29(5):218-23.

42. Khunti K, Gomes MB, Pocock S, Shestakova MV, Pintat S, Fenici P, et al. Therapeutic inertia in the treatment of hyperglycaemia in patients with type 2 diabetes: A systematic review. Diabetes Obes Metab. 2017. 
43. Ogura M, Harada-Shiba M. Clinical Inertia in the Management of Hypercholesterolemia: What Clinicians Need to do. J Atheroscler Thromb. 2016;23(5):552-3.

44. Siso Almirall A. Clinical inertia in osteoarthritis. Aten Primaria. 2012;44(2):72-3.

45. Kobelt G, Berg J, Lindgren P, Fredrikson S, Jönsson B. Costs and quality of life of patients with multiple sclerosis in Europe. Journal of Neurology, Neurosurgery \&amp; Psychiatry. 2006;77(8):918-26.

46. Gongora-Ortega J, Segovia-Bernal Y, Valdivia-Martinez Jde J, Galaviz-DeAnda JM, Prado-Aguilar CA. Educational interventions to improve the effectiveness in clinical competence of general practitioners: problem-based versus critical reading-based learning. BMC Med Educ. 2012;12:53.

47. Kennedy T, Regehr G, Rosenfield J, Roberts SW, Lingard L. Exploring the gap between knowledge and behavior: a qualitative study of clinician action following an educational intervention. Acad Med. 2004;79(5):386-93.

48. Djulbegovic B, Beckstead JW, Elqayam S, Reljic T, Hozo I, Kumar A, et al. Evaluation of Physicians' Cognitive Styles. Med Decis Making. 2014;34(5):627-37.

49. Kostopoulou O, Sirota M, Round T, Samaranayaka S, Delaney BC. The Role of Physicians' First Impressions in the Diagnosis of Possible Cancers without Alarm Symptoms. Med Decis Making. 2017;37(1):9-16.

50. Mamede S, van Gog T, van den Berge K, Rikers RM, van Saase JL, van Guldener C, et al. Effect of availability bias and reflective reasoning on diagnostic accuracy among internal medicine residents. JAMA. 2010;304(11):1198-203.

51. Saposnik G, Maurino J, Sempere AP, Terzaghi MA, Ruff CC, Mamdani M, et al. Overcoming Therapeutic Inertia in Multiple Sclerosis Care: A Pilot Randomized Trial Applying the Traffic Light System in Medical Education. Front Neurol. 2017;8:430.

52. van Hooff JC, van Buuringen M, El M'rabet I, de Gier M, van Zalingen L. Disgust-specific modulation of early attention processes. Acta Psychologica. 2014;152:149-57.

53. Reach G. Clinical inertia, uncertainty and individualized guidelines. Diabetes Metab. 2014;40(4):241-5.

54. Dijkstra IS, Pols J, Remmelts P, Brand PL. Preparedness for practice: a systematic cross-specialty evaluation of the alignment between postgraduate medical education and independent practice. Med Teach. 2015;37(2):153-61.

55. Monrouxe LV, Grundy L, Mann M, John Z, Panagoulas E, Bullock A, et al. How prepared are UK medical graduates for practice? A rapid review of the literature 2009-2014. BMJ Open. 2017;7(1):e013656.

56. Kostopoulou O, Russo JE, Keenan G, Delaney BC, Douiri A. Information distortion in physicians' diagnostic judgments. Med Decis Making. 2012;32(6):831-9.

57. Klucken T, Schweckendiek J, Koppe G, Merz CJ, Kagerer S, Walter B, et al. Neural correlates of disgustand fear-conditioned responses. Neuroscience. 2012;201:209-18.

58. Mataix-Cols D, An SK, Lawrence NS, Caseras X, Speckens A, Giampietro V, et al. Individual differences in disgust sensitivity modulate neural responses to aversive/disgusting stimuli. European Journal of Neuroscience. 2008;27(11):3050-8.

59. Otto B, Misra S, Prasad A, McRae K. Functional overlap of top-down emotion regulation and generation: An fMRI study identifying common neural substrates between cognitive reappraisal and cognitively generated emotions. Cognitive, Affective, \& Behavioral Neuroscience. 2014;14(3):923-38.

60. Sokol-Hessner P, Camerer CF, Phelps EA. Emotion regulation reduces loss aversion and decreases amygdala responses to losses. Social Cognitive and Affective Neuroscience. 2013;8(3):341-50.

61. M. de Melo C, Gratch J, Carnevale P. Humans vs. Computers: Impact of Emotion Expressions on People's Decision Making2014. 1- p.

62. van 't Wout M, Kahn RS, Sanfey AG, Aleman A. Affective state and decision-making in the Ultimatum Game. Experimental Brain Research. 2006;169(4):564-8. 


\section{SUPPLEMENTAL MATERIAL}

\section{Mediation Analysis:}

The mediation analysis characterizes the relationship between the independent variable (X; ambiguity aversion) and variables that are related with an outcome of interest ( $\mathrm{Y} ; \mathrm{TI})$. A mediating variable (M; facial muscle activation or emotional expression) is hypothesized to be intermediate in the relation between an independent variable $(\mathrm{X})$ and an outcome $(\mathrm{Y})$

\section{Figure e1. Schematic representation of the mediation analysis}

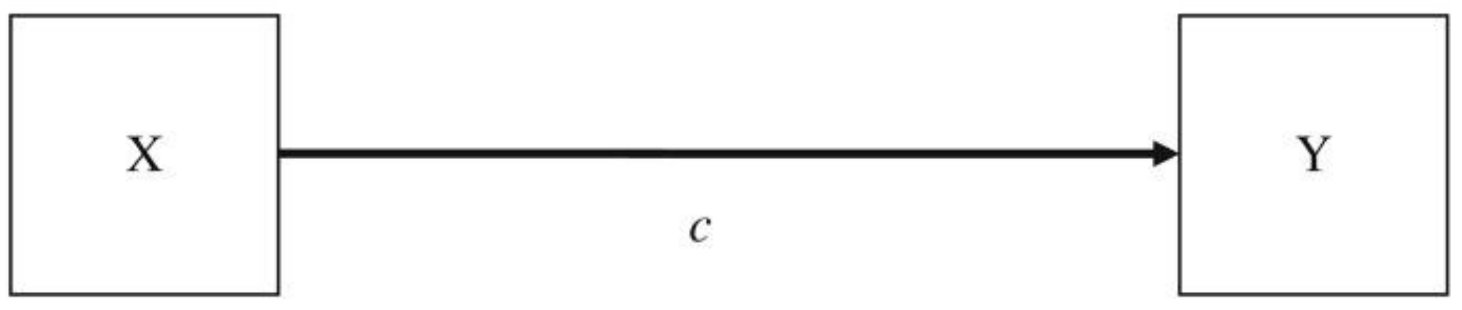

\section{(Total Effect of X on Y)}

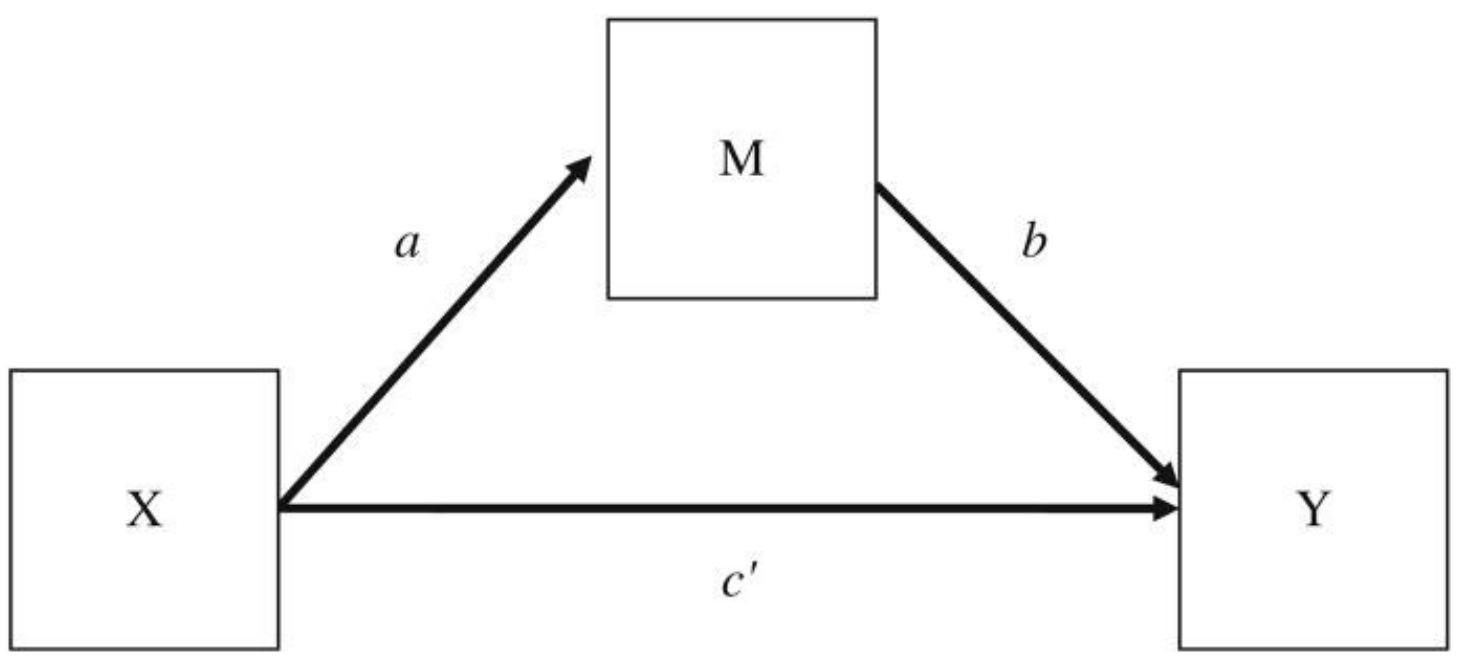

The mediation model decomposes the total effect of $X$ on $Y(c)$, into two parts: the indirect effect of $X$ on $Y$, quantified by $a b$ (the product of $a$ and $b$ ), and the direct effect of $X$ on $Y$ with the effect of the mediator removed, quantified by $c^{\prime}$; where $\mathrm{X}=$ aversion to ambiguity, $\mathrm{Y}=$ therapeutic inertia (TI) and $M=$ facial muscle activation or emotional expression. 
Figure e2. Template Graph illustrating the represented values following the structural equation modelling

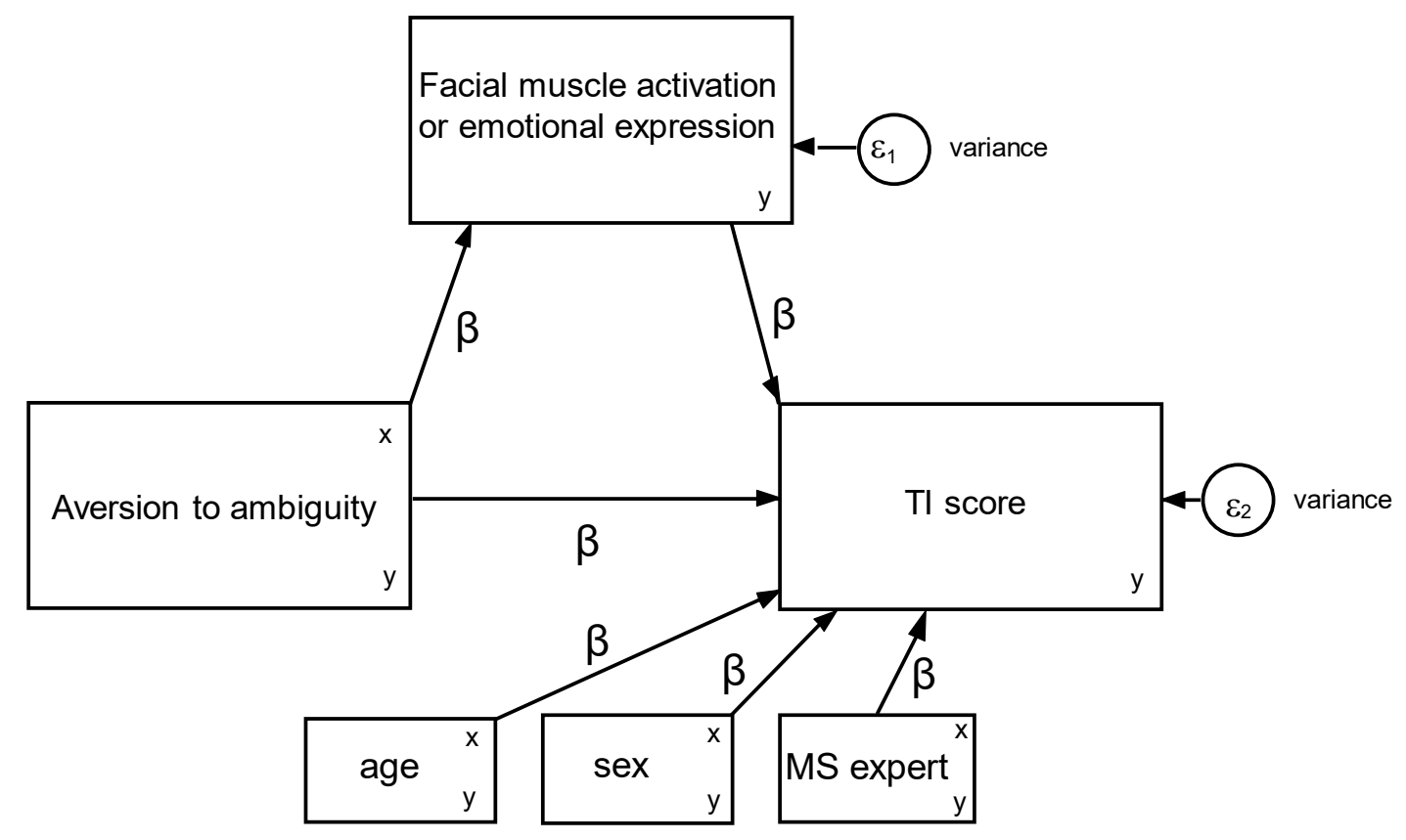

\section{Sensitivity analysis:}

The sensitivity analysis revealed no significant differences in the direction of the effect or relationship between ambiguity aversion and TI. For example, the inclusion of years of practice in the adjustment (instead of MS expertise) revealed that the total mediated effect of brow furrow was $8.6 \%(95 \% \mathrm{Cl} 5.2 \%$ $24.6 \%)$. Similarly, the total direct effects of disgust was $-8.3 \%(95 \% \mathrm{Cl}-5.0 \%$ to $-22.7 \%)$. Figures e3 and e4 (appendix) showed the minimal changes in the $\beta$ coefficients for the direct effect of aversion to ambiguity compared to figure 4 . There was a minimal increment $(<3 \%)$ to the R-square value $(R$-square: 0.34) when adding a facial or emotional variable into the mixed models with adjustment for years of practice instead of participants' expertise in MS care. Similar findings were observed when including participants volume of MS practice per week or practice settings (data not shown). 
Figure e3: Graphs representing the relationships between ambiguity aversion and the TI score in the sensitivity analysis with brow furrow as the mediator.

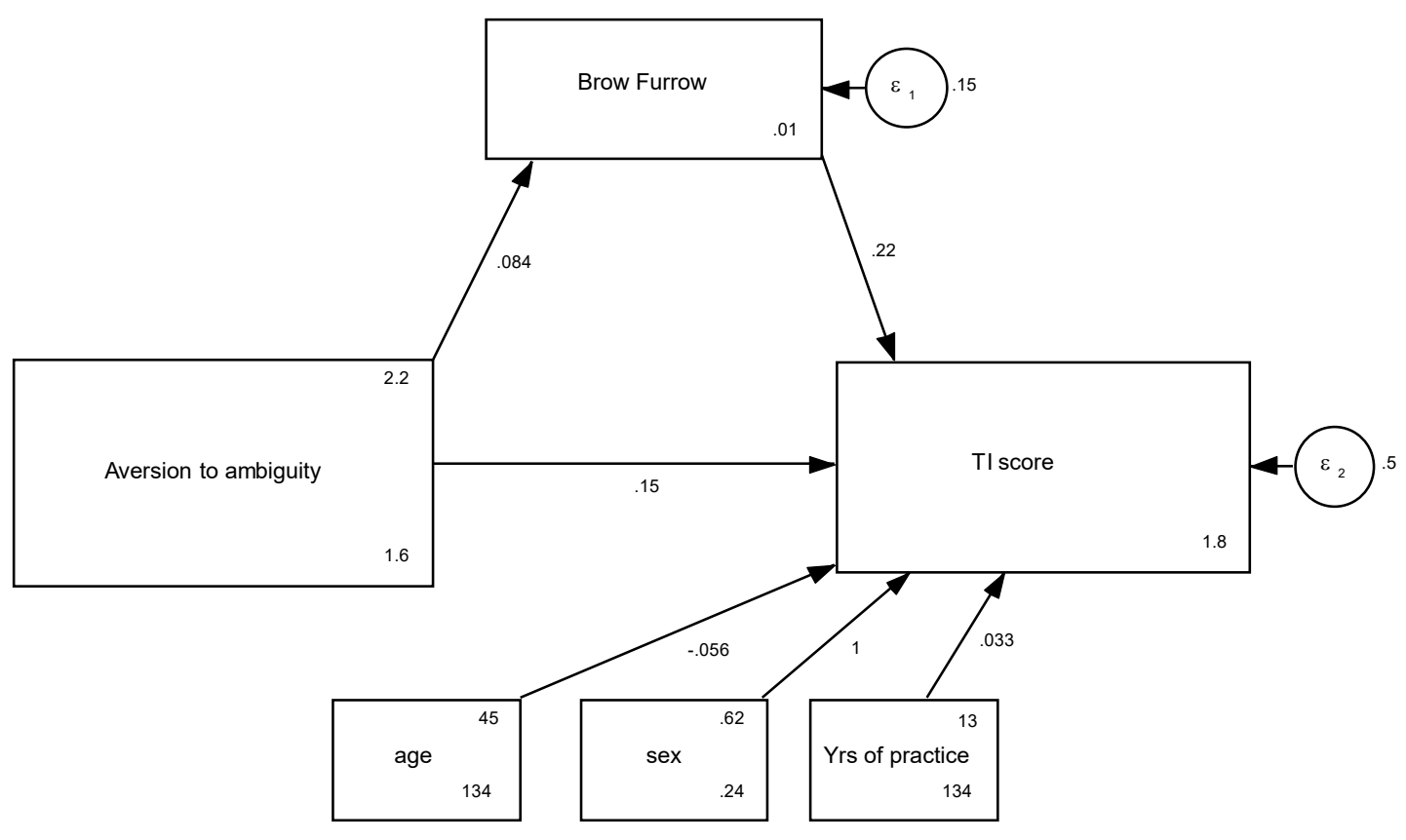

Figure e4: Graphs representing the relationships between ambiguity aversion and the $\mathrm{Tl}$ score in the sensitivity analysis with disgust as the mediator.

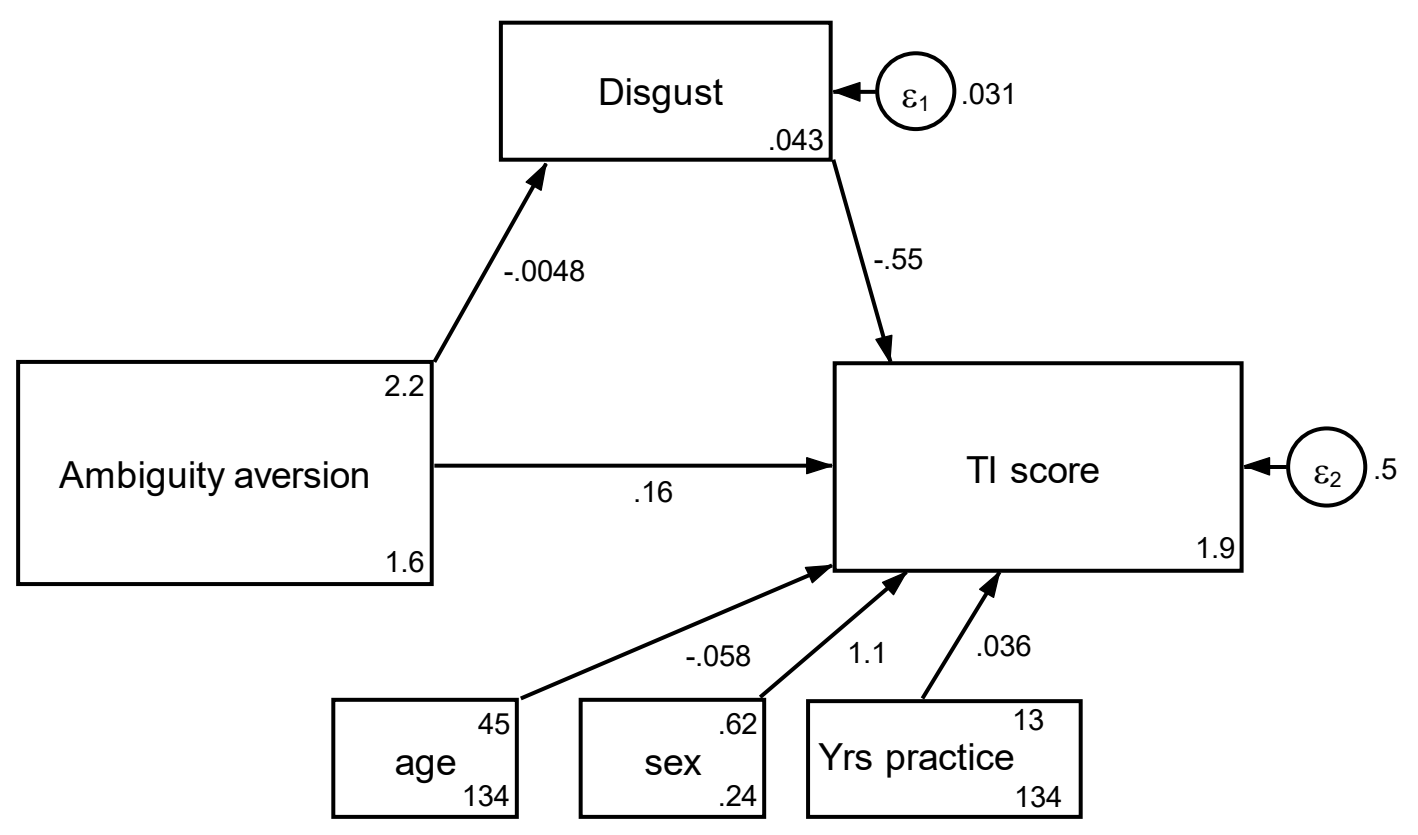

